\title{
Mixing parameterization: Impacts on rip currents and wave set-up
}

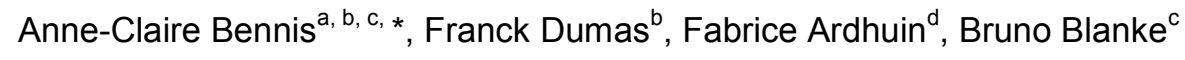 \\ ${ }^{a}$ M2C, UMR 6143 CNRS-UCBN-UR, 24 rue des Tilleuls, 14000 Caen, France \\ ${ }^{\mathrm{b}}$ DYNECO/PHYSED, Ifremer, Technopole de la pointe du diable, 29280 Plouzané, France \\ c LPO, UMR 6523 CNRS-Ifremer-IRD-UBO, 6 avenue Le Gorgeu, 29200 Brest, France \\ d LOS, Ifremer, Technopole de la pointe du diable, 29280 Plouzané, France \\ *: Corresponding author : Anne-Claire Bennis, tel.: +33 231565718 ; fax: +33 231565757 ; \\ email address : anne-claire.bennis@unicaen.fr
}

\begin{abstract}
:
Wave set-up is often underestimated by the models (e.g. Raubenheimer et al., 2001). Our paper discusses how the wave set-up may be changed by the inclusion of turbulent mixing in the bottom shear stress. The parameterization developed in Mellor (2002) for phase-averaged oscillatory boundary layer is used for this purpose. Two studies are carried out. The dependence of the parameterization on the vertical discretization and on the magnitude of the near-bottom wave orbital velocity is investigated. The function that distributes the turbulent terms over the vertical is modified, giving a good agreement with the average of the phase-resolved velocities, but an overestimation of the turbulent phase-resolved velocities. Applying that parameterization to simulate laboratory conditions in the presence of rip currents gives accurate magnitudes of the rip velocity, particularly in a fully coupled wave-current configuration, with an RMS error of about $4 \%$. Compared to a model using the more standard Soulsby (1995) parameterization, the wave set-up is increased by about $12 \%$ when using the alternative parameterization. Thus the bottom shear stress is sensitive to the mixing parameterization with a possible effect of turbulence on the wave set-up. Further measurement and parameterization efforts are necessary for practical applications.
\end{abstract}

\section{Highlights}

We study the impact of the mixing on the bottom friction and on the wave setup. A 1D study is performed. The original parameterization of Mellor (2002) is improved. The wave set-up is then increased by $12 \%$ for 3D simulations in nearshore.

Keywords: Bottom friction ; Vertical mixing ; Wave set-up ; Nearshore processes 


\section{Introduction}

Waves in the nearshore zone drive morphodynamic and hydrodynamic responses at many spatial and temporal scales (e.g. Svendsen, 2006). The most obvious hydrodynamic features are longshore currents (Bowen, 1969) and a mean sea level increase on the shore face (e.g. Longuet-Higgins and Stewart, 1963). Longuet-Higgins (1970) models the bottom shear stress as a linear combination of the alongshore current, the near-bottom orbital velocity and the bottom friction coefficient. As opposed to that, friction is believed to be a secondary term in the cross-shore momentum balance in which the wave-induced momentum flux divergence is mostly balanced by the hydrostatic pressure gradient associated with the wave set-up (e.g., Apotsos et al., 2007). An accurate parameterization of friction is thus the first priority when modeling flows in a surf zone. Many in situ experiments tried to determine a physical roughness parameter and various studies aimed at estimating meaningful friction coefficients from observed flow patterns (Feddersen et al., 2000 and Feddersen et al., 2003). These studies suggest that friction may not only be a function of bottom roughness, but also depend on wave breaking. Other sources of discrepancy between roughness and friction coefficients may stem from differences in roughness between the alongshore and cross-shore directions, because of specific form drags over bedforms (e.g. Barrantes and Madsen, 2000), and from the multiple velocity time scales that must be accounted when investigating the effect of bottom friction on either of the flow components (e.g., the wave effects on the dissipation of infragravity waves as in 
Reniers et al., 2002).

${ }_{47}$ Several studies (e.g. Raubenheimer et al., 2001; Apotsos et al., 2007) reported an 48 underestimation by the models of the wave set-up, in particular in depths shallower 49 than about one meter. So, our purpose here is to investigate a parameterization 50 of wave breaking effects on bottom friction, which impacts the wave set-up, by 51 adding breaking-induced turbulence to the phase-averaged mixing scheme proposed 52 by Mellor (2002, hereafter referred to as ML02) for modeling the bottom boundary 53 layer. The parameterization uses turbulent kinetic energy to represent the influ54 ence of wave-induced near-bottom turbulence on the mean flow, and was shown to 55 accurately reproduce the observed current profiles in the case of an oscillatory bot56 tom boundary layer (Mellor, 2002). We extend its use by assessing its performance 57 in another modeling framework and focusing on its ability to reproduce nearshore 58 hydrodynamics.

59 In section 2, we redo the validation case presented in Mellor (2002) for a one60 dimensional oscillatory flow superimposed to a mean flow, to validate our implemen${ }_{61}$ tation of the ML02 parameterization. Tests in presence of wave breaking are also 62 performed. In section 3, the mixing parameterization is evaluated for a nearshore sit${ }_{63}$ uation with rip currents. The ML02 results are tested against the laboratory data of 64 Haas and Svendsen (2002). A comparison with the Soulsby (1995) parameterization 65 is also performed. Conclusions follow in section 4 .

\section{${ }_{66} 2$ Oscillatory bottom boundary layer}

${ }_{67}$ We investigate the effects of vertical mixing on the bottom shear stress with the 68 mixing parameterization proposed by Mellor (2002). The same equations and forcing conditions as in the original paper of Mellor are used. Our experiment describes the oscillation of the bottom boundary layer with the wave phase for a one-dimensional 
72

73 First, we compare phase-averaged simulations obtained with the mixing parameter-

74 ization, with phase-resolving simulations, for a non-breaking case. Next, we study

75 the behavior of the parameterization in presence of wave breaking.

\section{${ }_{76} \quad 2.1 \quad$ Methodology}

77 We use the MARS hydrodynamical model (Lazure and Dumas, 2008), with some

modifications to simulate a one-dimensional vertical case. In MARS, the pressure projection method is implemented to solve the unsteady Navier-Stokes equations under the Boussinesq and hydrostatic assumptions. The model uses the ADI (Alternate Direction Implicit) time scheme according to Bourchtein and Bourchtein (2006). Finite difference schemes are used for the spatial discretization, which is done on an Arakawa-C grid.

The equations of motion for a horizontally forced, one-dimensional vertical, incompressible, unsteady flow are

$$
{ }_{86} \begin{aligned}
\frac{\partial u}{\partial t} & =\frac{\tau_{0 x}}{h}+\lambda u_{b x} \omega \cos (\omega t)+\frac{\partial \tau_{x}}{\partial z}, \\
\frac{\partial k}{\partial t} & =\underbrace{\frac{1}{D^{2}} \cdot \frac{\partial}{\partial \varsigma}\left(\frac{\nu_{V}}{s_{k}} \cdot \frac{\partial k}{\partial \varsigma}\right)}_{=\operatorname{Diff}}+\mathrm{B} \underbrace{-\epsilon}_{=\text {Diss }}+\underbrace{\mathrm{P}+\mathcal{P}_{k}}_{=\text {Prod }}, \\
\frac{\partial \epsilon}{\partial t} & =\frac{1}{D^{2}} \cdot \frac{\partial}{\partial \varsigma}\left(\frac{\nu_{V}}{s_{\epsilon}} \cdot \frac{\partial \epsilon}{\partial \varsigma}\right)+\frac{\epsilon}{k}\left(c_{1} \operatorname{Prod}+c_{3} \text { Buoy }-c_{2} \epsilon\right)+\mathcal{P}_{\epsilon} .
\end{aligned}
$$

where $u$ is the flow velocity in the x-direction, $k$ is the turbulent kinetic energy (hereafter TKE), $\epsilon$ is the turbulent dissipation, $D$ is the mean depth and $h=D / 2, \varsigma$ is the terrain-following coordinate and $t$ is the time. The term $\tau_{x}$ is the $\mathrm{x}$-component of the Reynolds stress. When we consider the phase-resolving solution, all quantities described in equations (2.1), (2.2), (2.3) depend on the wave phase (with $\lambda=1$ in eq. (2.1)), the forcing terms depend on time and all phases are simulated. The wave 
95 phase is given by $\Phi=\frac{360^{\circ} \times t}{T}$ (where $T$ is the wave period set to $9.6 \mathrm{~s}$ as in Mellor's

96 study). For phase-averaged simulations, all quantities described in equations (2.1),

97 (2.2), (2.3) are phase-averaged (with $\lambda=0$ in eq. (2.1)) and the forcing terms

115

116

$$
\begin{aligned}
\mathcal{P}_{k}= & \underbrace{\alpha \frac{4 D_{w}}{H_{r m s}}\left(1-\frac{2 z^{\prime}}{H_{r m s}}\right)_{z^{\prime} \leq z_{r e f}}}_{=\mathcal{P}_{k b}}+\underbrace{\beta \omega\left|\mathbf{u}_{b}\right|^{2}\left(F_{1 \psi} F_{2 z}\right)^{3}}_{=\mathcal{P}_{k f}} \\
\mathcal{P}_{\epsilon}= & \underbrace{1.44\left(\alpha \frac{\epsilon}{k}\right)\left[\left(\frac{4 D_{w}}{H_{r m s}}\left(1-\frac{2 z^{\prime}}{H_{r m s}}\right)_{z^{\prime} \leq z_{r e f}}\right)\right]}_{=\mathcal{P}_{\epsilon b}}+\underbrace{\beta \frac{\epsilon}{k}\left[C \omega\left|\mathbf{u}_{b}\right|^{2}\left(F_{1 \psi} F_{2 z}\right)^{3}\right]}_{=\mathcal{P}_{\epsilon f}}
\end{aligned}
$$

${ }_{117}$ where $F_{1 \Psi}$ and $F_{2 z}$ are given in Mellor (2002) (see his equations (18),(20) and (21a)). 
$F_{1 \Psi}$ accounts for the angle between the waves and the current. $F_{2 z}$ distributes the source terms over the water column and therefore depends on depth. $F_{2 z}$ is also a function of the bottom roughness $\left(z_{0}\right) . z_{0}$ is set to $3 \cdot 06 \cdot 10^{-5} \mathrm{~m}$ to keep only the terms $0.0488+0.02917 l z+0.01703 l z^{2}$ in $F_{2 z} . C$ is a non-dimensional constant equal to $0.9337 .\left|\mathbf{u}_{b}\right|$ is the magnitude of the orbital velocity such as $\left|\mathbf{u}_{b}\right|=\left(u_{b x}^{2}\right)^{1 / 2}$. $z_{\text {ref }}$ is the distribution length for the dissipation due to wave breaking $\left(D_{w}\right)$. The wave dissipation is computed with the help of the friction velocity $\left(u_{\star}\right)$ such as $D_{w}=\alpha^{\prime} u_{\star}^{3}$, with $\alpha^{\prime}=100$ (Craig and Banner, 1994). $u_{\star}$ is the water friction velocity. $H_{r m s}$ is the root mean square significant wave height. $z^{\prime}$ is the distance from the surface. Four situations are discussed:

a) phase-averaged solution without breaking wave $(\alpha=0, \beta=1)$.

b) phase-averaged solution with breaking wave $(\alpha=1, \beta=1)$.

c) phase-resolving solution without breaking wave $(\alpha=0, \beta=0)$.

d) phase-resolving solution with breaking wave $(\alpha=1, \beta=0)$.

The coefficients $\alpha$ and $\beta$ are chosen to combine the turbulent source terms introduced by Walstra (2000) and Mellor (2002). The input of TKE resulting from wave breaking is distributed over the water column as in Rascle et al. (2013), who highlighted the efficiency of this modeling strategy, and not injected at the surface (e.g. Feddersen and Trowbridge (2005), Burchard (2001)).

Aside from the previous equations, the formulation of the bottom shear stress must be modified to account for the wave effects. For the phase-averaged solution, the ML02 formulation uses near-bottom TKE such as:

$$
\tau_{b x}=\frac{u \kappa S_{M 0} \sqrt{2 k_{0}}}{\ln \left(\frac{z_{b}}{z_{0}}\right)}, \quad z_{b}>z_{0},
$$

and 


$$
\tau_{b x}=\frac{u \kappa S_{M 0} \sqrt{2 k_{0}}}{\ln \left(\frac{z_{b}}{z_{0}}+1\right)}, \quad 0<z_{b} \leq z_{0}
$$

143

$$
\epsilon_{\text {surf }}=\frac{u_{\star}^{3}}{\kappa\left(z^{\prime}+z_{0}^{s}\right)}\left[a+\left(\frac{3 \sigma_{k}}{2}\right)^{1 / 2} C_{\mu}^{1 / 4} C_{w}\left(\frac{z^{\prime}+z_{0}^{s}}{z_{0}^{s}}\right)^{-m}\right],
$$

where $\tau_{b x}$ is the x-component of the bottom shear stress, $z_{b}$ is the first grid point above the bottom, $k_{0}$ is the TKE near the bottom, $\kappa$ is the Von Kármán constant set to $0.4, z$ is the distance above the bottom and $S_{M 0}$ is a stratification parameter taken equal to 0.39 for a neutral flow.

We have for the phase-resolving solution:

$$
\tau_{b x}=\left(\frac{u \kappa}{\ln \left(\frac{z_{b}}{z_{0}}\right)}\right)^{2} \text {, if } z_{b}>z_{0} \text {, and } \tau_{b x}=\left(\frac{u \kappa}{\ln \left(\frac{z_{b}}{z_{0}}+1\right)}\right)^{2} \text {, if } 0<z_{b} \leq z_{0} \text {. }
$$

With wave breaking, the boundary conditions for TKE and dissipation are changed. At the surface, we prefer the dirichlet boudary conditions of Kantha and Clayson (2004), based on the friction velocity, instead of Walstra et al (2000). Then, we have:

$$
k_{\text {surf }}=\frac{1}{2} B_{1}^{2 / 3} u_{\star}^{2}\left[1+3 m b \alpha^{\prime}\right]^{2 / 3}
$$

where the constants $B_{1}, m, b$ are equal to $16.64,1,0.2210$, respectively, and

where $k_{\text {surf }}$ and $\epsilon_{\text {surf }}$ are the surface value of the turbulent kinetic energy and of the dissipation, respectively. The constants $a, \sigma_{k}, C_{\mu}, C_{w}$ are equal to $1,1,0.09,100$ respectively. $z_{0}^{s}$ is the surface roughness. The expression of $z_{0}^{s}=0.6 \cdot H_{s}$, given by Terray et al. (1996), is used. 

phase-resolving solution.

\begin{tabular}{lr}
\hline Characteristic & Value \\
\hline Water depth & $2 h=4 \mathrm{~m}$ \\
Wave frequency & $\omega=0.65 \mathrm{rad} / \mathrm{s}$ \\
x-component of the near-bottom wave orbital velocity & $u_{b x}=2 \mathrm{~m} / \mathrm{s}$ \\
x-component of the mean wall shear stress & $\tau_{0 x}=0.004 \mathrm{~m}^{2} / \mathrm{s}^{2}$ \\
Model time step & $d t=0.04 \mathrm{~s}$ \\
\hline
\end{tabular}

Table 1: Parameters used in one-dimensional simulations.

171

\subsection{Experiments}

The main goal of the experimental plan is to assess the performance of the mixing parameterization in our modeling system. For this purpose, the second validation case shown in Mellor (2002) is repeated. Note that a validation for a pure oscillatory flow of Jensen et al. (1989) was carried out before this study, but it is not presented here for sake of conciseness. In this section, a fully developped mean flow superimposed on an oscillatory flow is chosen. We choose the same parameters as in the ML02 experiment. They are summarized in Table 1. A similar method is also chosen to validate our implementation: a phase-averaged solution is compared to a

First, we compare the vertical profiles of velocity, turbulent kinetic energy and turbulent dissipation obtained in both solutions. For the phase-resolving solution, a mean is taken over one wave period. Simulations with and without wave breaking are performed to evaluate how the flow is modified by wave breaking. These simulations are calculated at high resolution, with 1200 grid points. Second, we evaluate the flow sensitivity to the vertical mesh. Several meshes (all with 1200 grid points) refined near the bottom and the surface are employed. Moreover, simulations at low resolution are performed with 20 vertical grid points that are regularly distributed. A one-meter depth is used at low resolution whereas we choose a four-meter depth at high resolution. From these experiments, an expression for the $F_{2 z}$ function is derived. 


\section{$2.3 \quad$ Results}

\subsubsection{Phase-resolving vs. Phase-averaged}

Figure 2.1 compares the velocity profiles obtained in the phase-averaged and phaseresolving solutions. When wave breaking is not included (Figure 2.1, first panel), the vertical profile calculated by the mixing parameterization is very close to the phase-resolving solution. Near-bottom TKE values are greatly increased (by a factor of three) in phase-averaged calculations (see Figure 2.2, NO BREAK case: top and bottom panels) because the mixing parameterization uses an additional source term of TKE, maximum near the bottom. This term is essential to get the phase-averaged and phase-resolving solutions to coincide. It allows reducing the velocity and ensures that its vertical profile is in conformity with the reference. The high bottom value of TKE is reminiscent of the difficulties encountered with mixing length models for the simulation of the air flow over waves (Miles, 1996). Indeed, the oscillations due to waves are known to prevent turbulent mixing when the eddy overturning time becomes larger than the wave period (Belcher and Hunt, 1993). Under these conditions, the classical mixing length models generally fail to reproduce this effect and overestimate mixing in the outer boundary layer (Miles, 1996), especially when they are applied to the phase-averaged flow. The turbulent dissipation is maximum near the bottom in absence of wave breaking (Figure 2.3).

To ensure that our computations for turbulent kinetic energy are correct, we compare for each wave phase our vertical profiles with the ones given by Jensen et al. (1989) and by Mellor (2002). Note that this comparison is done for a pure oscillatory flow with a depth of 28 centimeters. Our TKE agrees with the laboratory data of Jensen (1989) and with the TKE computed by Mellor's model (Figure 2.4). Near the bottom, a similar problem to Mellor's simulations is observed: TKE is overestimated. This is probably due to the modeling framework that seems inappropriate to represent the flow measured in a U-tube. 

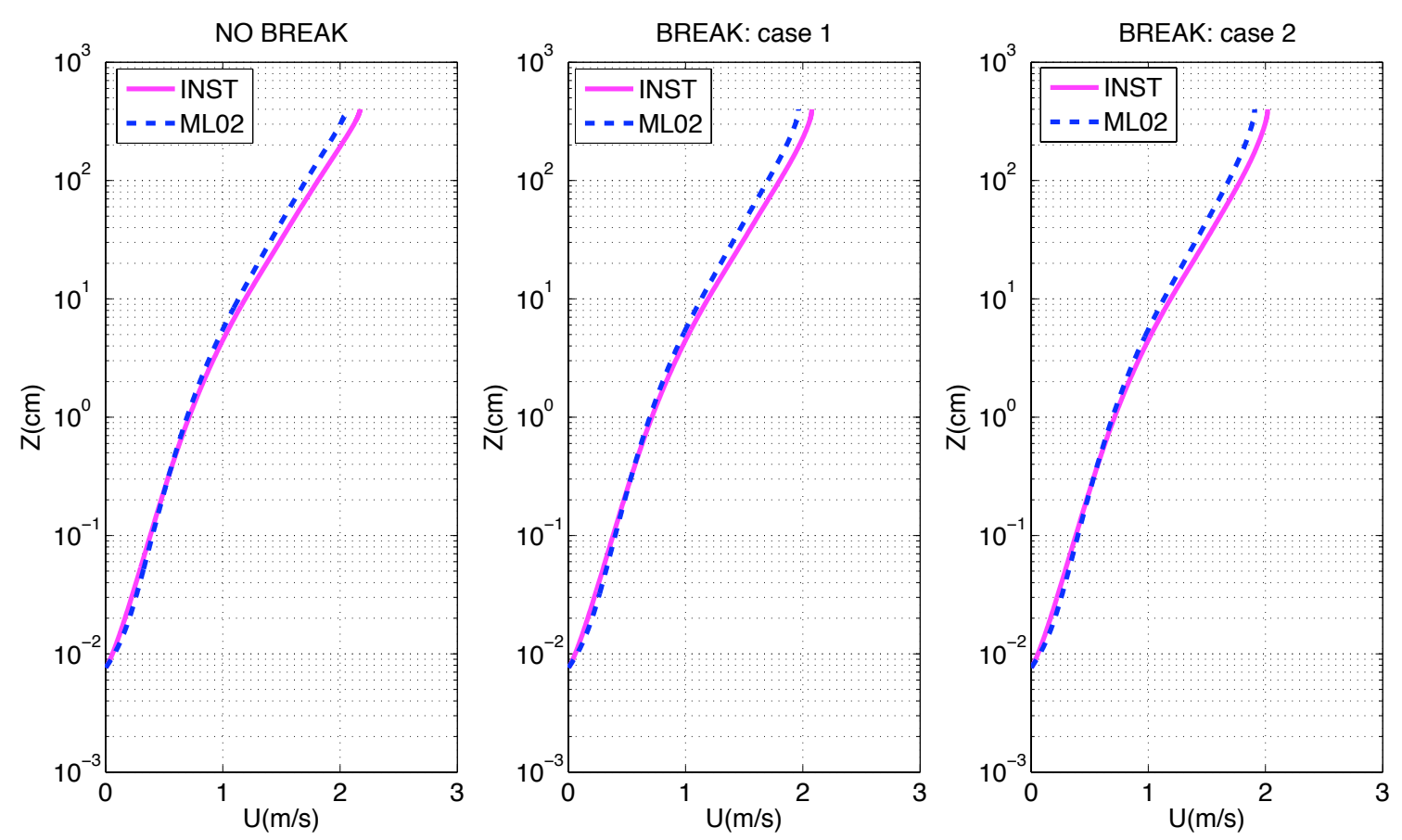

Figure 2.1: Vertical profiles of the velocity. INST: Phase-resolving case. ML02: Phase-averaged case. NO BREAK: Case without wave breaking. "BREAK: case 1" and "BREAK: case 2" labels refer to breaking cases obtained with $z_{\text {ref }} \simeq 1 \mathrm{~m}$ and $z_{\text {ref }} \simeq 3 \mathrm{~m}$, respectively.

We now evaluate the performance of the mixing parameterization in presence of wave breaking. Indeed, our goal is to use it for nearshore applications where the waves break. This configuration was not addressed in the original paper of Mellor. The effects of wave breaking are parameterized. The additional mixing induced by breaking is introduced according to Walstra et al. (2000) (see equations (2.4) and (2.5) with $\alpha=1$ ). Note that the additional source term of TKE is computed from a phase-averaged solution, which is appropriate for this case. Since the phase-averaged profiles are computed by an arithmetic average of the instantaneous profiles, we inject TKE at each phase in the phase-resolving solutions. The McCowan-type criterion is used to estimate the significant wave height. We test two characteristic lengths to distribute the breaking-induced turbulent source terms (see $z_{\text {ref }}$ value in equations (2.4) and (2.5)). Our goal is to study the behavior of ML02 for different $z_{\text {ref }}$ because this parameter is not always set to $H_{r m s} / 2$ as advocated in Walstra 
(2000) and must be changed according to the studied case. We use the following lengths: $z_{r e f}=H_{r m s} / 2 \simeq 1 \mathrm{~m}\left(\right.$ as in Walstra, 2000) and $z_{r e f}=11 H_{r m s} / 8 \simeq 3 \mathrm{~m}$. Both source terms depend on wave energy dissipation resulting from wave breaking, such that $D_{w}=6.75 .10^{-4} \mathrm{~m}^{3} \cdot \mathrm{s}^{-3}$ (and $\rho_{0} D_{w}=0.69 \mathrm{~W} \cdot \mathrm{m}^{-2}$, where $\rho_{0}$ is the reference water density set to $1027 \mathrm{~kg} \cdot \mathrm{m}^{-3}$ ). The friction velocity computed by Alves and Banner (2003) is used to estimate wave energy dissipation. Feddersen and Trowbridge (2005) showed that only a fraction of wave energy dissipation is related to breaking. Here, we intentionally inject the totality of the dissipation so that breaking effects are accentuated. To consider the effects of wave breaking, the boundary conditions at the surface are modified according to equations (2.9) and (2.10). For both characteristic lengths, the turbulence of wave breaking does not penetrate down to the bottom of the water column. Therefore, the near-bottom TKE is not modified (see Figure 2.2, BREAK: cases 1 and 2) and is still overestimated by the mixing parameterization. In comparison with the NO BREAK case, wave breaking homogenizes TKE over most of the water column. Moreover, as the depth-integrated value of the source terms is the same for both cases with wave breaking, the vertical profiles of TKE are almost similar. The depth-integrated TKE in case 2 is about $0.9 \%$ greater than in case 1, most probably because of numerical effects induced by the refined vertical mesh. With a non-refined mesh, the depth-integrated TKE would be the same for both cases. Figure 2.5 shows the TKE budget over the vertical: the production (Prod) and diffusion (Diff) terms balance the dissipation (Diss) term. When a steady state is reached, equation (2.2) becomes:

$$
0=\text { Diff }+ \text { Prod }+ \text { Diss }
$$

Since the dissipation term is negative, because it is homogeneous to $-\epsilon$, it balances the other terms (Diff and Prod). Besides TKE production by shear, the production terms include the sources related to wave breaking and to ML02. The mixing induced by wave breaking reduces the vertical shear and slows down the flow lo- 
cally. The deeper the penetration of mixing, the smaller the surface velocity (see 249 Figure 2.1, BREAK: cases 1 and 2). However, in both present cases, the effects of ${ }_{250}$ wave breaking on the velocity are weak. The wave breaking process increases the 251 turbulent dissipation near the surface and the ML02 solution agrees the reference 252 solution (Figure 2.3, BREAK: cases 1 and 2). Altogether, the mixing parameteriza253 tion works well in presence of wave breaking at the surface: the phase-averaged and 254 phase-resolving profiles show very close results. 

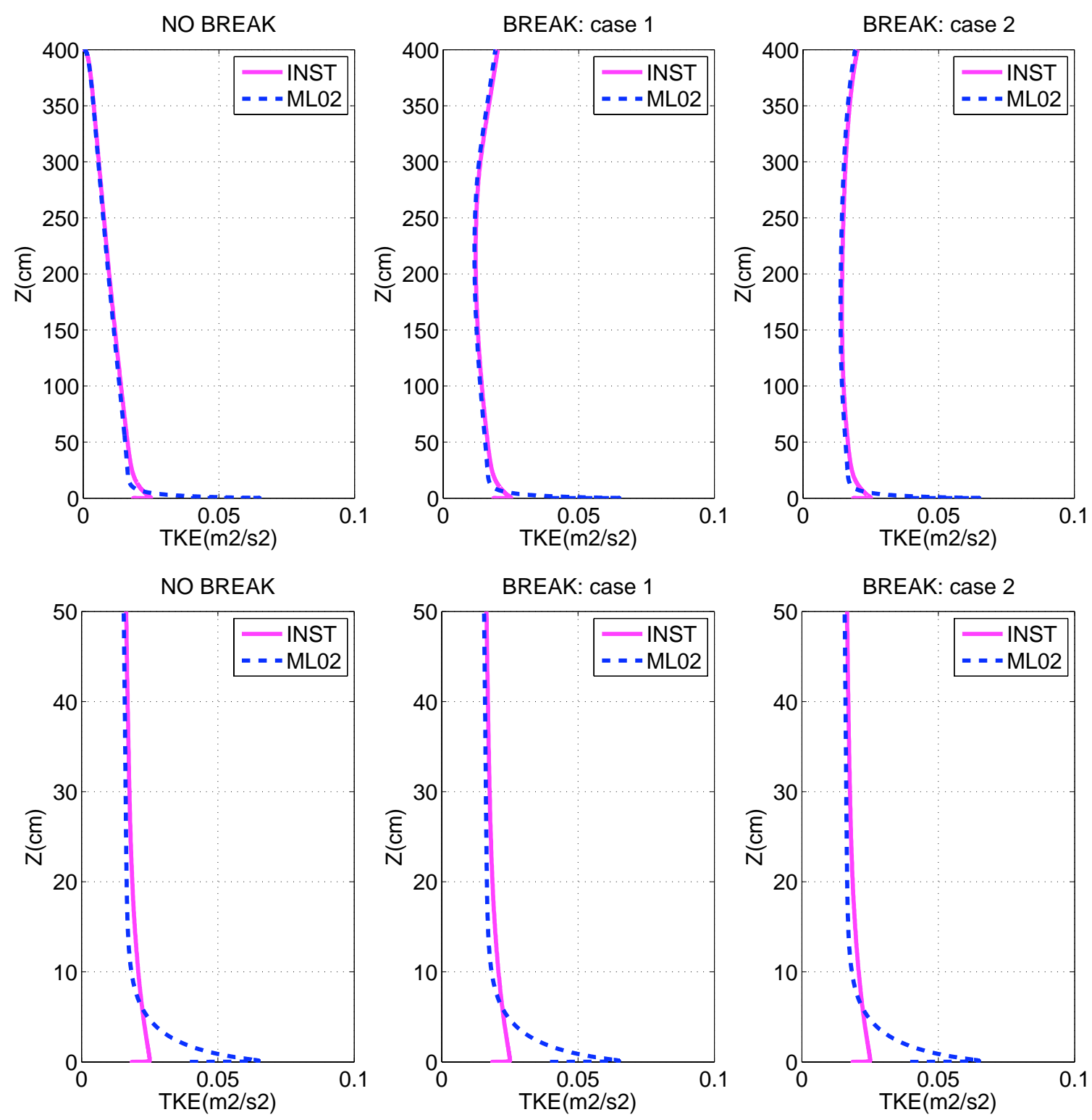

Figure 2.2: Vertical profiles of TKE for the non-breaking case (NO BREAK) and the breaking case with different distributions of wave breaking (BREAK: case 1, $z_{\text {ref }} \simeq 1 \mathrm{~m}$ and BREAK: case $2, z_{\text {ref }} \simeq 3 \mathrm{~m}$ ). INST: Phase-resolving case. ML02: Phase-averaged case. Top panel: Entire water column. Bottom panel: zoom above the bottom 50 centimeters. 

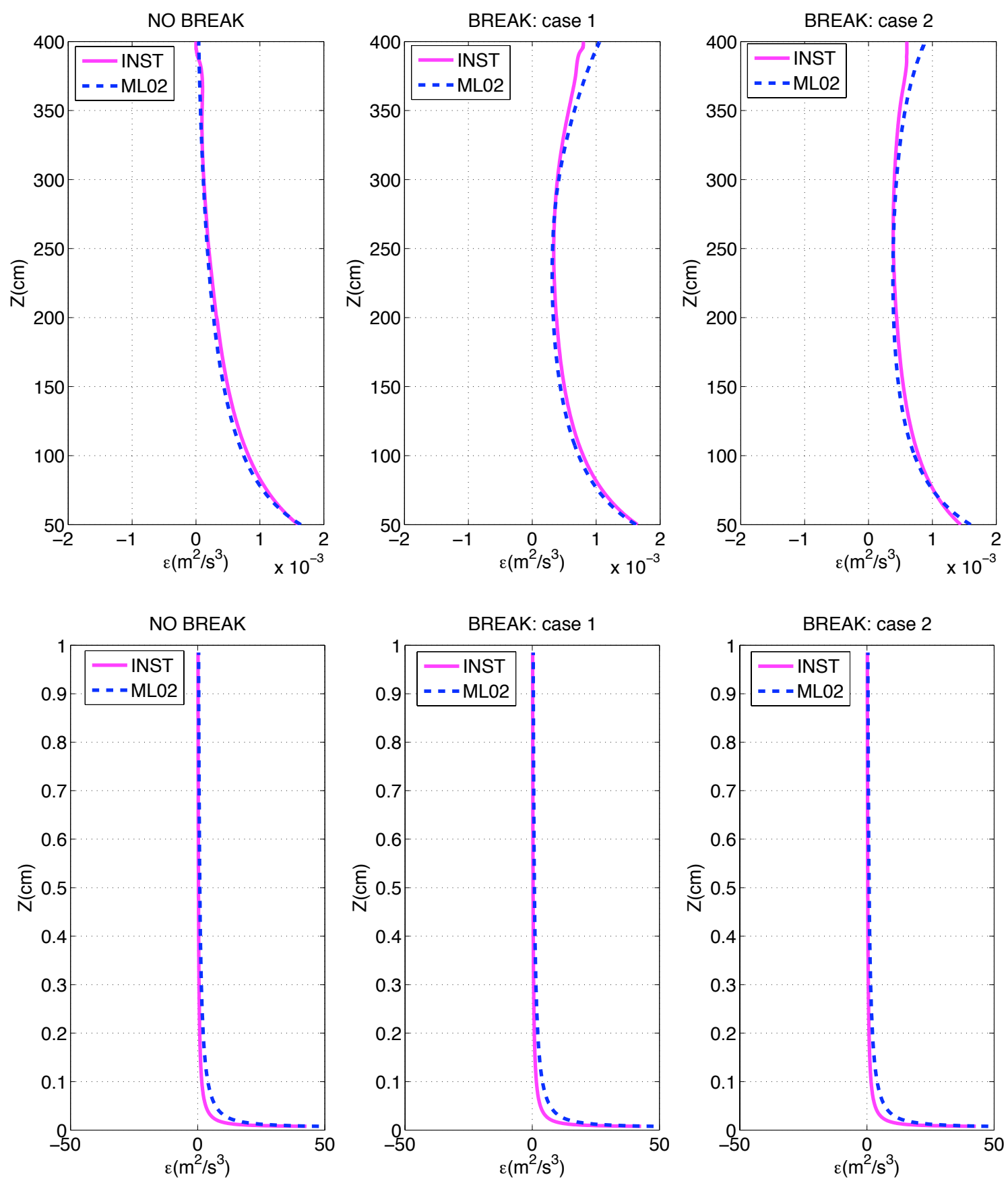

Figure 2.3: Vertical profiles of dissipation for the non-breaking case (NO BREAK) and the breaking case with different distributions of wave breaking (BREAK: case $1, z_{\text {ref }} \simeq 1 \mathrm{~m}$ and BREAK: case $2, z_{r e f} \simeq 3 \mathrm{~m}$ ). INST: Phase-resolving case. ML02: Phase-averaged case. The top row shows the entire water column down to a depth of fifty centimeters. The bottom row shows only the first centimeter. 

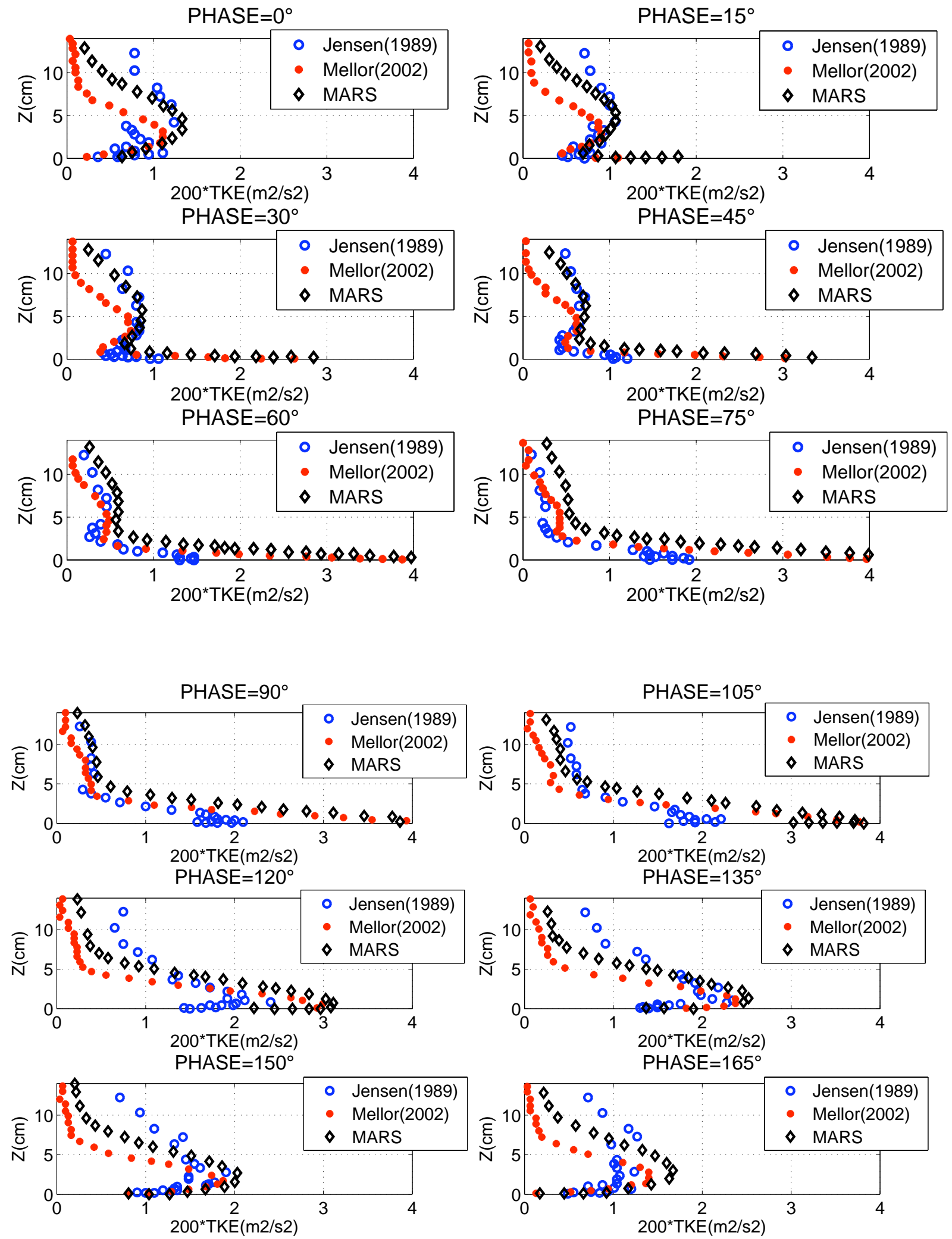

Figure 2.4: Pure oscillatory flow and phase-resolving case: comparison of vertical profiles of TKE for each wave phase with a 15-degree increment. Models results from MARS (black diamonds) and POM used in Mellor (2002) (red circles). Data of Jensen et al (1989) are in blue circles. The flow for the phases from 180 to 360 degrees is a mirror image of the one shown here. 

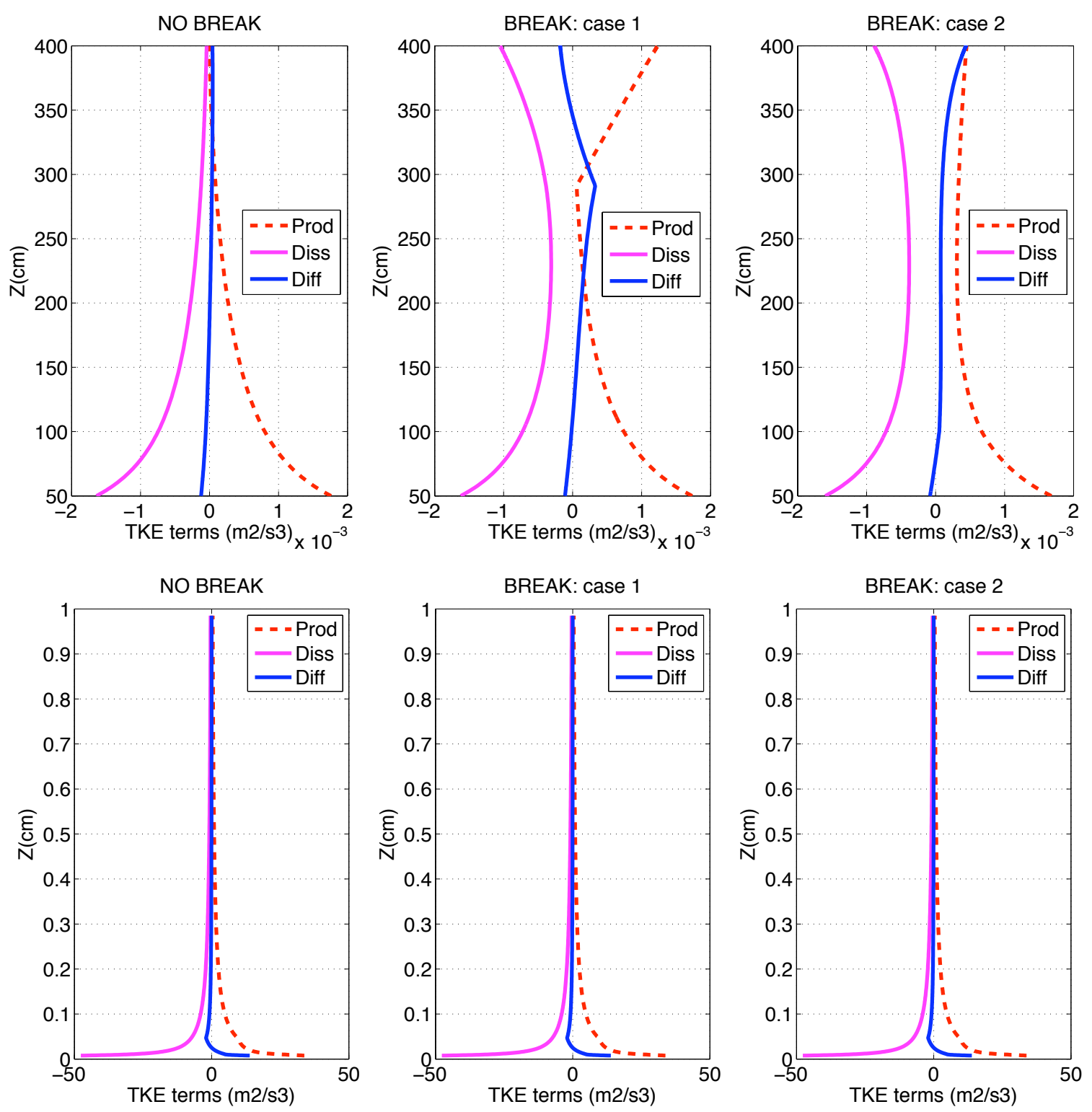

Figure 2.5: TKE budget for ML02. The production (Prod), dissipation (Diss) and diffusion (Diff) terms are plotted as a function of depth and their expression is given in equation (2.2). The top row shows the entire water column down to a depth of fifty centimeters. The bottom row shows only the first centimeter. The NO BREAK, BREAK: case 1, BREAK: case 2 labels refer to the non-breaking case, the breaking case for $z_{\text {ref }} \simeq 1 \mathrm{~m}$ and the breaking case for $z_{\text {ref }} \simeq 3 \mathrm{~m}$, respectively. 


\subsection{2 $F_{2 z}$ function}

The formula for the $F_{2 z}$ function strongly affects the solution given by the mixing parameterization. Shape and magnitude of the velocity, TKE and turbulent dissipation are modified. Mellor derived a formula to fit with the phase-resolving solution. His function is:

$$
F_{2 z}=\gamma_{1}+\gamma_{2} \cdot \ln \left(\frac{z \omega}{\left|\mathbf{u}_{\mathbf{b}}\right|}\right)+\gamma_{3} \cdot\left[\ln \left(\frac{z \omega}{\left|\mathbf{u}_{\mathbf{b}}\right|}\right)\right]^{2}
$$

where $\gamma_{1}, \gamma_{2}, \gamma_{3}$ are constants and set to $-0.0488,0.02917,0.01703$, respectively (more details in Appendix A). The other terms of $F_{2 z}$ are zero because of the value of the bottom roughness set to $z_{0}=3.06 \times 10^{-5} \mathrm{~m}$, which removes the term: $5+$ $\log _{10}\left(\frac{z_{0} \omega}{\left|\mathbf{u}_{\mathbf{b}}\right|}\right)$. It is easy to remark the dependence of $F_{2 z}$ on both the depth and the wave orbital velocity.

When $z \rightarrow 0, \ln \left(\frac{z \omega}{\left|\mathbf{u}_{\mathbf{b}}\right|}\right)$ tends to infinity. Then also $F_{2 z}$ goes to infinity. To illustrate this, five differently refined meshes are tested (more details in Appendix A). The depth of the grid point nearest the bottom $\left(z_{b o t}\right)$ differs according to the mesh. $F_{2 z}$ near the bottom is strongly affected by $\left(z_{b o t}\right)$ and here varies from 0.2 to 5.5 (Figure 2.6). The near-bottom value of $F_{2 z}$ modifies the shape of the vertical profile of the velocity. The smaller the value, the more reduced the vertical shear, whereas the velocity profile for the phase-resolving case keeps the same shape. After many numerical experiments, we derived a new $F_{2 z}$ function, named $F_{2 z, \text { mod }}$ :

$$
F_{2 z, \text { mod }}=\|\mathcal{A}\|-\frac{\ln (N)}{3 \sqrt{N}}
$$

$273 \quad$ with: $\mathcal{A}=\frac{p_{1} \cdot \ln (N)}{\sqrt{N}} \cdot(\ln (l z) \cdot l z)^{2}$ and $l z=\ln \left(\frac{z \omega}{\left|\mathbf{u}_{b}\right|}\right)-p_{2}$

${ }_{274} N$ is the total number of grid points and $\|\cdot\|$ is the complex norm. $p_{1}$ and $p_{2}$ are constants and set to 0.0028 and 0.38 , respectively. The new function also goes to infinity when $\mathrm{z}$ tends to zero but grows up more slowly and, therefore, allows the 277 use of the smallest values of $z_{\text {bot }}$. 
We clip all negatives values to only add turbulent source terms, as recommended 279 by Mellor (2002). Note that the depth-integrated value of $F_{2 z}$ is modified for the 280 different meshes when these negative values are clipped.
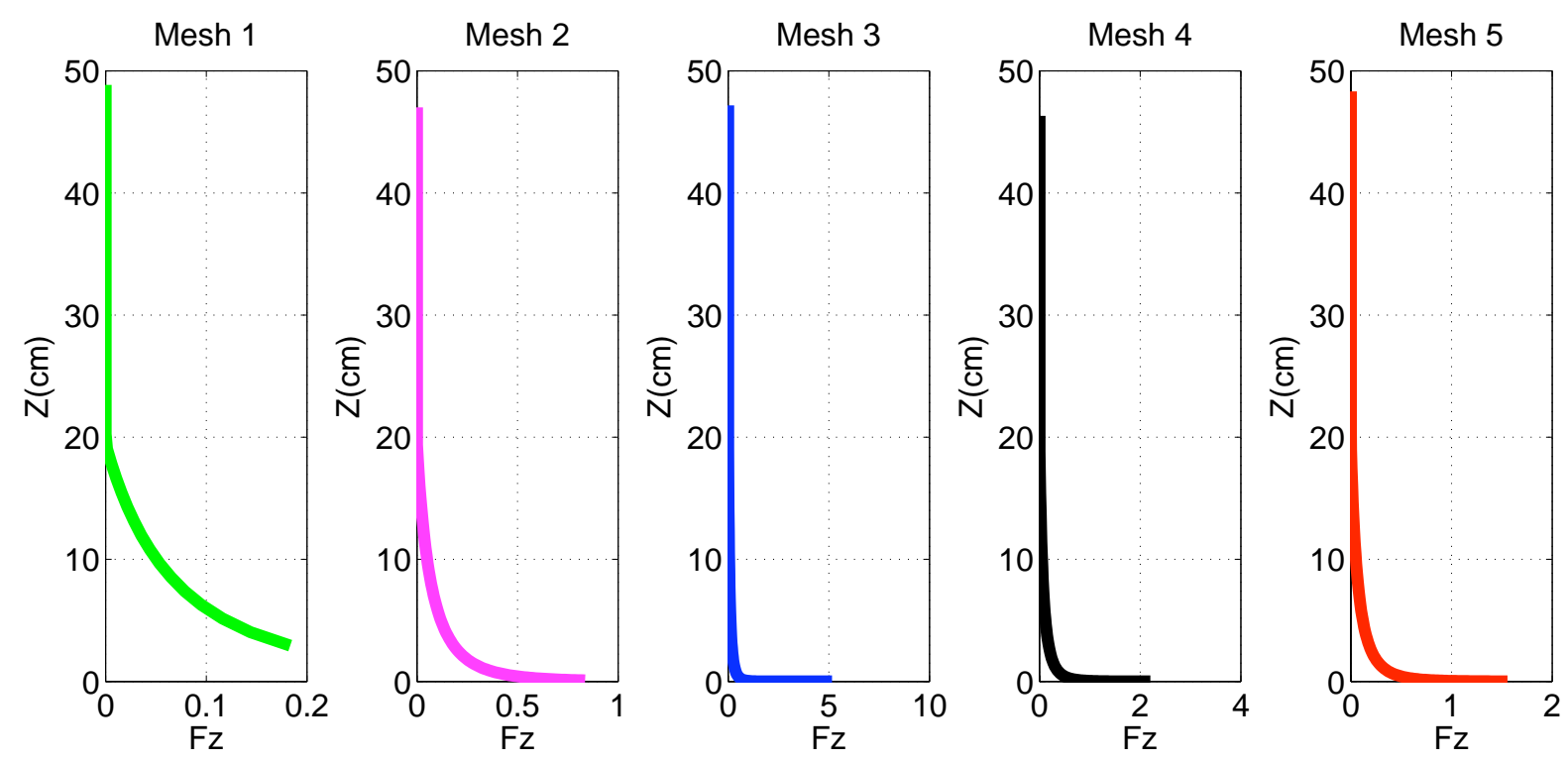

Figure 2.6: Near-bottom zoom of $F_{2 z}$ for all meshes (bottom 50 centimeters).

${ }_{281}$ Figures 2.1 shows that the magnitude and the shape of the phase-averaged velocity

282

283

profile agree with the phase-resolving ones. We also test another mesh, whose resolution is low, like the one used in operational applications. This mesh counts 20 vertical grid points and is regular. The depth now is one meter. The vertical profiles of the velocity, TKE and dissipation for both the non-breaking and breaking cases are shown in Figure 2.7. Profiles with the new function are referred to 'ML02 (b)' while 'ML02 (a)' refers to the profiles obtained with the original function. Clearly, the formula for $F_{2 z}$ is crucial to allow fit with the phase-resolving reference solution. When this function is not appropriate like in 'ML02 (a)', the shape and the magnitude of the velocity are not correct. Moreover, near-bottom TKE is too weak. The velocity profiles obtained with the new function agree with the phase-resolving ones for both the BREAK and NO BREAK cases. The impact of wave breaking is more significant than before because the depth is shallower. As explained before, the near-bottom TKE had to be increased to obtain correct velocities. Therefore, 
an overestimation of near-bottom TKE is also observed here. As a coarser mesh is used, this overestimation goes up to the first twenty centimeters, while that problem is confined near the bottom at high resolution.

We also diagnose the influence of the near-bottom wave orbital velocity on the results produced by the mixing parameterization (ML02). As discussed in the previous section, near-bottom values of the $F_{2 z}$ function may change according to the vertical mesh and lead to numerical inaccuracy. When $\left|\mathbf{u}_{b}\right|$ goes to zero, both the $F_{2 z}$ and $F_{2 z, \text { mod }}$ functions produce positive values near the surface because they both tend to infinity. These positive values introduce turbulent source terms near the surface, which is not physically realistic because the functions should be maximum near the bottom and zero at the surface. From now on, we remove all unrealistic positive values of the functions near the surface, besides their negative values.

To sum up, the mixing parameterization has been adapted successfully for use in our modeling platform after a new $F_{2 z}$ function was derived. The mixing parameterization with this function works well at high resolution but also at low resolution. The performances in presence of wave breaking are acceptable. 

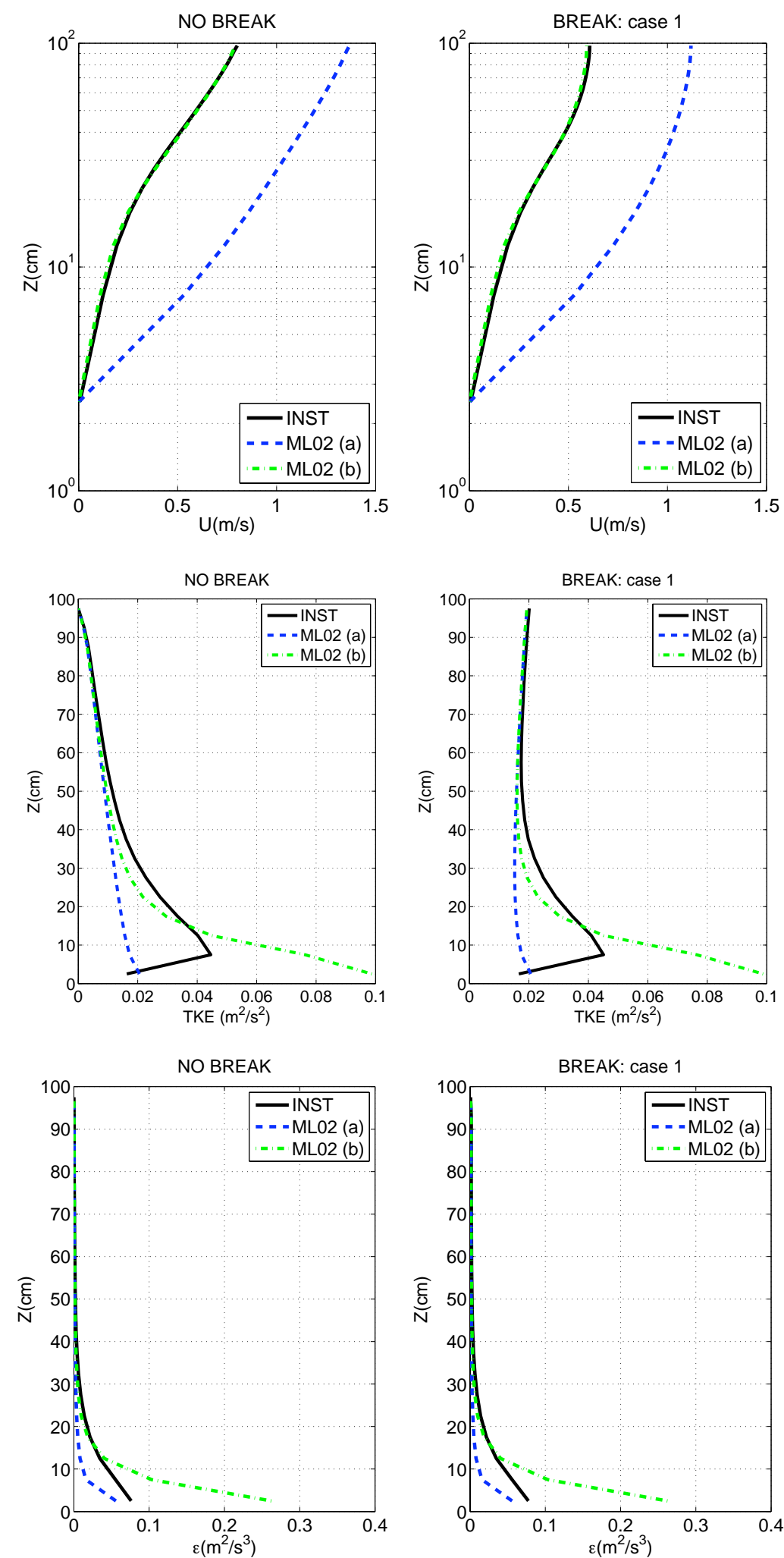

Figure 2.7: Vertical profiles of velocity (top row), TKE (middle row) and dissipation (bottom row). INST: Phase-resolving case. ML02 (a): Phase-averaged case with the original $F_{2 z}$ function. ML02 (b): Phase-averaged case with the modified $F_{2 z}$ function NO BREAK: Case without wave breaking. BREAK: case 1 refer to the breaking case for $z_{r e f} \simeq 1 \mathrm{~m}$. 


\section{Nearshore application}

The mixing parameterization is now used nearshore and tested against laboratory data of Haas and Svendsen (2002). Comparisons with Soulsby'95 parameterization are also performed. We want to highlight how the use of the mixing parameterization changes the simulation of the wave set-up.

\subsection{Methodology}

Numerical experiments are carried out with the fully coupled three-dimensional wave-current model: MARS-WAVEWATCH III (Bennis et al., 2011). The modeling platform uses an automatic coupler (PALM) that allows us to combine MARS3D and WAVEWATCH III (see Figure 3.1). Two coupling options are available: oneway or two-way modes. In the one-way mode, the feedback of the currents on the waves is not included in the computation (see black arrows in Figure 3.1), unlike in the two-way mode (black and gray arrows in Figure 3.1). The results given by both coupling modes are compared. Indeed, some recent studies still use only the one-way mode.

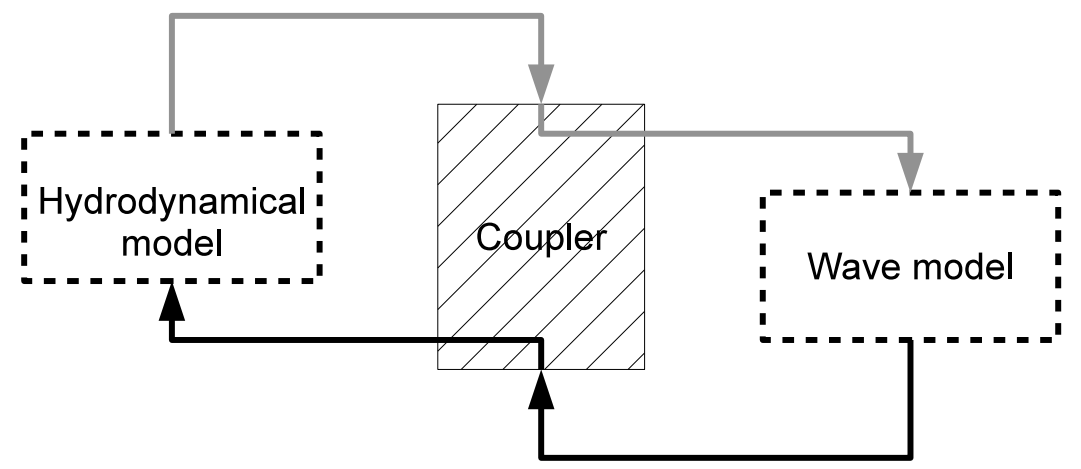

Figure 3.1: Coupling procedure. The black arrows refer to the one-way mode while the whole set of black and gray arrows shows to the two-way mode. The wave model is WAVEWATCH III, the hydrodynamical model is MARS3D and the coupler is PALM.

The momentum equations of the hydrodynamical model (MARS3D) are based on 
the quasi-Eulerian velocity (Ardhuin et al. (2008) and Bennis et al. (2011)):

$$
\frac{D \widehat{U}}{D t}=\widehat{\mathbf{F}}_{\mathbf{E P G}}+\widehat{\mathbf{F}}_{\mathrm{VM}}+\widehat{\mathbf{F}}_{\mathbf{H M}}+\widehat{\mathbf{F}}_{\mathrm{BA}}+\widehat{\mathbf{F}}_{\mathrm{BBL}}+\widehat{\mathbf{F}}_{\mathbf{V F}}+\widehat{\mathbf{F}}_{\mathrm{WP}}
$$

where $\widehat{\mathbf{U}}=(\widehat{u}, \widehat{v}, \widehat{w})$ is the quasi-Eulerian velocity vector, $\widehat{\mathbf{F}}_{\mathbf{E P G}}$ is the pressure gradient, $\widehat{\mathbf{F}}_{\mathbf{V M}}$ and $\widehat{\mathbf{F}}_{\mathbf{H M}}$ represent the forces due to vertical and horizontal mixing, respectively, $\widehat{\mathbf{F}}_{\mathbf{B A}}$ is the breaking acceleration, $\widehat{\mathbf{F}}_{\mathbf{B B L}}$ represent forces caused by the streaming, $\widehat{\mathbf{F}}_{\mathbf{V F}}$ is the vortex force and $\widehat{\mathbf{F}}_{\mathbf{W P}}$ is the wave-induced pressure gradient. Equations (3.1) are able to reproduce the three-dimensional circulation in the presence of the waves. These equations are validated for adiabatic cases (e.g. Bennis et al , 2011) and for cases with dissipation representative of nearshore conditions (e.g. Moghimi et al. (2012)). They are similar to the set of equations of McWilliams et al. (2004) that has been largely validated for nearshore applications (e.g. Uchiyama et al (2010), Kumar et al (2012)). The standard k- $\epsilon$ turbulent scheme is used to model the vertical turbulence. The surface boundary conditions are changed to account for the mixing due to wave breaking: the schemes are Kantha and Clayson (1994) for TKE and Craig (1996) for dissipation. The model of Walstra (2000) is employed for the vertical distribution of turbulence in the water column, except at the surface where the previous schemes are preferred to ensure better results. The wave energy dissipation resulting from wave breaking and bottom friction is linearly distributed over a length set to $H_{r m s} / 2$ for breaking and over the thickness of the wave bottom boundary layer $(\delta)$ for bottom friction. $\delta$ is computed as:

$$
\delta=\frac{2 \kappa}{\sigma}\left|\mathbf{u}_{\mathrm{orb}}\right| \sqrt{\frac{f_{w}}{2}},
$$

where $\sigma$ is the intrinsic wave radian frequency, $\mathbf{u}_{\text {orb }}$ is the near-bottom wave orbital and $f_{w}$ is the friction factor according to Soulsby (1995). $f_{w}$ is defined as: 


$$
f_{w}=1.39\left[\left(\frac{\sigma z_{0}}{\left|\mathbf{u}_{\text {orb }}\right|}\right)^{0.52}\right],
$$

where $z_{0}$ is the bottom roughness which is set to five millimeters in the next. The wave energy dissipation due to wave breaking is computed by the wave model while the dissipation due to the bottom stress is obtained by the following relation:

$$
D_{f}=\frac{1}{2 \sqrt{\pi}} f_{w}\left|\mathbf{u}_{\mathbf{o r b}}\right|^{3} .
$$

2 The spectral wave model, WAVEWATCH III, is phase-averaged. The transport equation of the wave action density spectrum $\mathcal{N}(\mathcal{N}$ being a function of time, space, wave number and direction) is used to simulate the wave propagation. Wave physics is accounted by some source and sink terms that are included in the right handside of the transport equation. They represent wind-wave interaction, non-linear wave-wave interactions, linear input, dissipation by whitecapping, wave-bottom interaction, depth-induced breaking and bottom scattering (for more details, see Tolman, 2009). As we use a phase-averaged wave model, the expression of the bottom shear stress must account for the oscillations of the wave bottom boundary layer with the wave phase. Therefore, the use of the mixing parameterization seems very wise. Standard parameterizations are based on the near-bottom wave orbital velocity. Soulsby (1995) parameterization (hereafter SB95) is one of them and we will compare it to the mixing parameterization (ML02).

\subsection{Experiments}

We use laboratory data of Haas and Svendsen (2002), provided to us by K. Haas (personal communication), to test our simulations. The bathymetry (see Figure 3.2 ) is stretched by a factor of twenty as explained in Kumar et al. (2012). The domain is extended by $108 \mathrm{~m}$ in both the cross-shore and longshore directions to avoid interference with the boundary conditions (BC). We obtain a cross-shore width 
of $312 \mathrm{~m}$ and an alongshore length of $568 \mathrm{~m}$. Periodic BCs are used at the lateral boundaries, whereas open boundary conditions (OBC) and no-slip conditions are used offshore and onshore, respectively. The horizontal grid resolution is set to $4 \mathrm{~m}$ in each direction, for both the wave and hydrodynamical models. MARS3D uses 20 regular sigma levels over the vertical. This vertical discretization helps to minimize the computational cost. In the previous section, the ML02 parameterization has been tested with a similar discretization (more details in section 2.3.2). The time step is set to $0.5 \mathrm{~s}$ for both models and the coupling time step is equal to $1 \mathrm{~s}$.

Battjes (1975) shows that the horizontal viscosity is affected by wave breaking for 2DH configurations. We choose for our three-dimensional simulations to apply a constant horizontal viscosity coefficient everywhere. So, the vertical mixing is affected equally over the grid, since the vertical turbulence is the main subject of this study. Then, our conclusions will be to some extent independent of lateral mixing though, of course, horizontal mixing decreases the overall turbulence level. Furthermore, the three-dimensional effects redistribute the mixing due to wave breaking. The hydrodynamical model is forced by an incident wave of $1 \mathrm{~m}$ offshore. The peak period is set to $6.25 \mathrm{~s}$. The wave spectrum is Gaussian and the wave incidence is normal to avoid the development of an alongshore current, which could prevail over the rip current for an angle of incidence greater than $10^{\circ}$ (Weir et al., 2011). The wave model uses 36 directions and the directional resolution is thus set to $10^{\circ}$ as in Kumar et al. (2012). Twenty-five frequencies are used in the range of $0.04-1.1 \mathrm{~Hz}$. A depth-induced breaking constant $(\gamma)$ of 0.55 is used (Battjes and Janssen, 1978; Eldeberky and Battjes, 1996), which is close to the value of 0.6 used by Kumar et al. (2012) for the same experiment. A $\gamma$ value of 0.73 is also tested. This type of modeling for breaking allow us compare our results with those of Kumar et al. (2012), noting that more accurate parameterizations for the dissipation due to wave breaking have been recently proposed (e.g. Filipot et al. (2010) and Leckler et al. (2013)) 


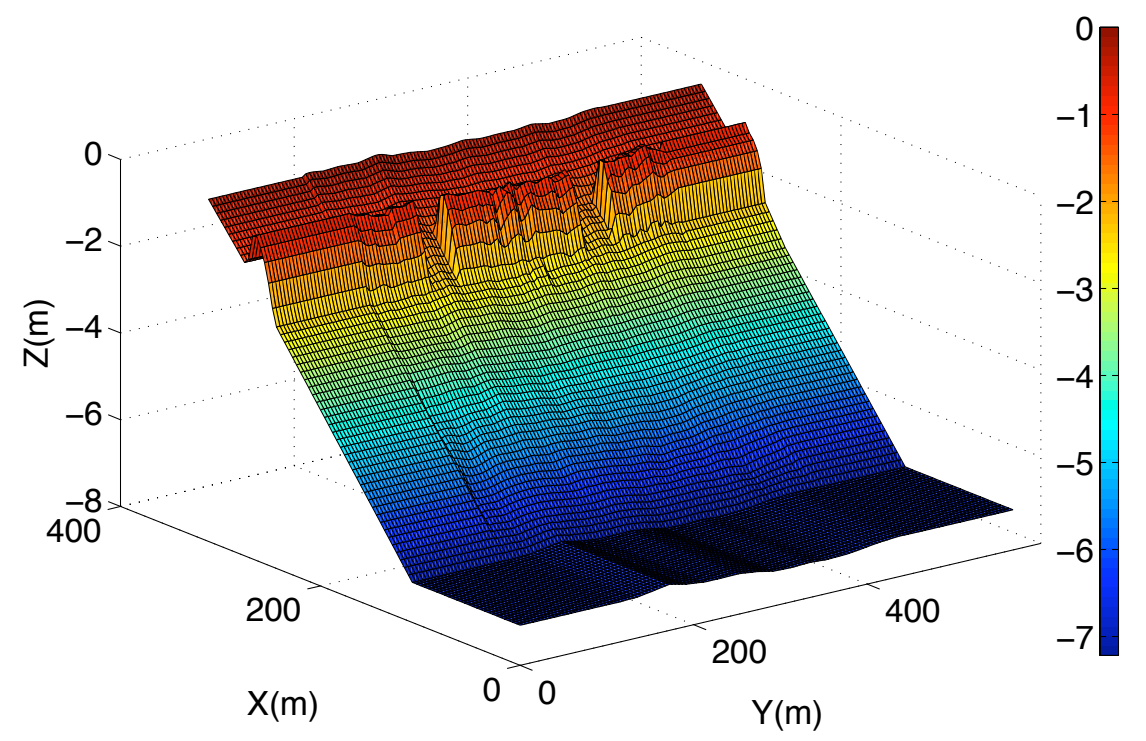

Figure 3.2: Bathymetry.

Both the ML02 and SB95 parameterizations are tested against the laboratory data.

Vertical profiles of the cross-shore velocity and cross-shore profiles of the significant wave height and mean sea surface elevation are examined. Results for both coupling modes are also compared. The influence of the $\gamma$ value is also evaluated. Table 2 summarizes the main parameters used in the simulations. Other details about the studied configurations are given in Table 3.

\begin{tabular}{lr}
\hline Characteristic & Value \\
\hline Wave height at the offshore & $1 \mathrm{~m}$ \\
Wave peak period at the offshore & $6.5 \mathrm{~s}$ \\
Wave breaking constant & 0.55 or 0.73 \\
Model time step & $0.5 \mathrm{~s}$ \\
Coupling time step & $1 \mathrm{~s}$ \\
Horizontal space grid & $4 \mathrm{~m}$ \\
Directional resolution & $10 \mathrm{deg}$ \\
\hline
\end{tabular}

Table 2: Parameters used in numerical simulations. 


\begin{tabular}{|c|c|c|c|}
\hline Cases & $\gamma$ & $\begin{array}{c}\text { Coupling } \\
\text { mode }\end{array}$ & $\begin{array}{c}\text { Bottom stress } \\
\text { parameterization }\end{array}$ \\
\hline C1 & 0.55 & two-way & SB95 \\
\hline C2 & 0.73 & one-way & SB95 \\
\hline C3 & 0.73 & two-way & SB95 \\
\hline C4 & 0.55 & two-way & ML02 \\
\hline C5 & 0.73 & one-way & ML02 \\
\hline C6 & 0.73 & two-way & ML02 \\
\hline
\end{tabular}

Table 3: Description of the studied cases that differ by the depth-induced breaking constant $(\gamma)$, the coupling mode and the bottom stress parameterization.

\subsection{Results}

\subsubsection{Rip velocity}

The vertical structure of the quasi-Eulerian rip velocity (named rip velocity here) is discussed in this section. Comparisons with data are performed for Test $\mathrm{R}$ (Haas and Svendsen, 2002), which corresponds to Test B of Haller et al. (2002). Here are the main results: (a) The rip current computed in the one-way mode is larger than the observations inside the channel for both parameterizations (see Figure 3.3). RMS errors of about $9 \%$ are found (see Table 4), instead of $2.5 \%$ in two-way. (b) The fully coupled (two-way mode) flow agrees well with the observations at all locations. The vertical structure of the velocity displays a similar shape as in Kumar et al. (2012). The rip velocity is maximum within the water column and decreases toward the surface and the bottom. This shape differs from the observations that suggest a maximum at the surface, though no near-surface measurements are available. The near-surface velocity would probably be improved with a roller model. (c) Offshore, the differences between the two coupling modes are smaller than inside the rip channel. The vertical profiles are almost similar (see Figure 3.3). (d) All parameterizations work well in the two-way mode and reproduce the channel flow.

They produce similar currents at all locations except near the bottom (see Figure 3.3). We discuss this point in the next section. (e) The $\gamma$ value has a little impact 
on the vertical structure of the cross-shore current.

Differences between the two coupling modes agree with the studies of Yu and Slinn (2003) and of Weir et al. (2011), although their conclusions were established from 2DH studies. They showed that the feedback compacts the rip current and reduces its offshore extension. This behavior is accentuated for the depth-integrated crossshore current and one can reasonably think that a similar behavior exists for the three-dimensional cross-shore current. Here, we notice that the cross-shore current is always weaker in two-way coupling and, therefore, its offshore extension is smaller. The impact of the two-way mode is intensified inside the rip channel because the current is strong at this location and modifies the wave fields due to the change in the wave number, in particular. Weir et al. (2011) also observe a reduction of the breaking acceleration due to the change in wave height.

\begin{tabular}{|c|c|c|c|c|c|}
\hline & $\mathbf{X}(\mathbf{m})$ & SB95f & SB95c & ML02f & ML02c \\
\hline Prof. 1 & 11.80 & $9 \%$ & $\mathbf{2 . 5} \%$ & $9 \%$ & $\mathbf{2 . 5} \%$ \\
\hline Prof. 2 & 11 & $6 \%$ & $3 \%$ & $5.5 \%$ & $\mathbf{2 . 5} \%$ \\
\hline Prof. 3 & 10.5 & $5 \%$ & $4 \%$ & $4 \%$ & $\mathbf{3} \%$ \\
\hline Prof. 4 & 10 & $4 \%$ & $4 \%$ & $\mathbf{3} \%$ & $\mathbf{3} \%$ \\
\hline Prof. 5 & 9.5 & $6 \%$ & $6 \%$ & $\mathbf{4 . 5 \%}$ & $\mathbf{4 . 5} \%$ \\
\hline Mean & all & $6 \%$ & $4 \%$ & $5 \%$ & $\mathbf{3 \%}$ \\
\hline
\end{tabular}

Table 4: Root mean square error (RMSE) for Test R. Minimum RMSE values are in bold. ML02f and ML02c refer to the mixing parameterization used for the one-way and the two-way mode, respectively. SB95f and SB95 refer to the parameterization proposed by Soulsby (1995) for the one-way and the two-way mode, respectively.

\subsubsection{Wave set-up}

We investigate the impact of the bottom shear stress parameterization on the wave set-up. The sensitivity to the depth-induced breaking constant and to the coupling mode is also studied. As the wave set-up is sensitive to the increasing of the wave height (e.g. Raubenheimer et al. (2001)), we test two values for the depth induced breaking constant $(\gamma)$. The values of 0.55 (Nelson (1994), Nelson (1997)) and of 0.73 

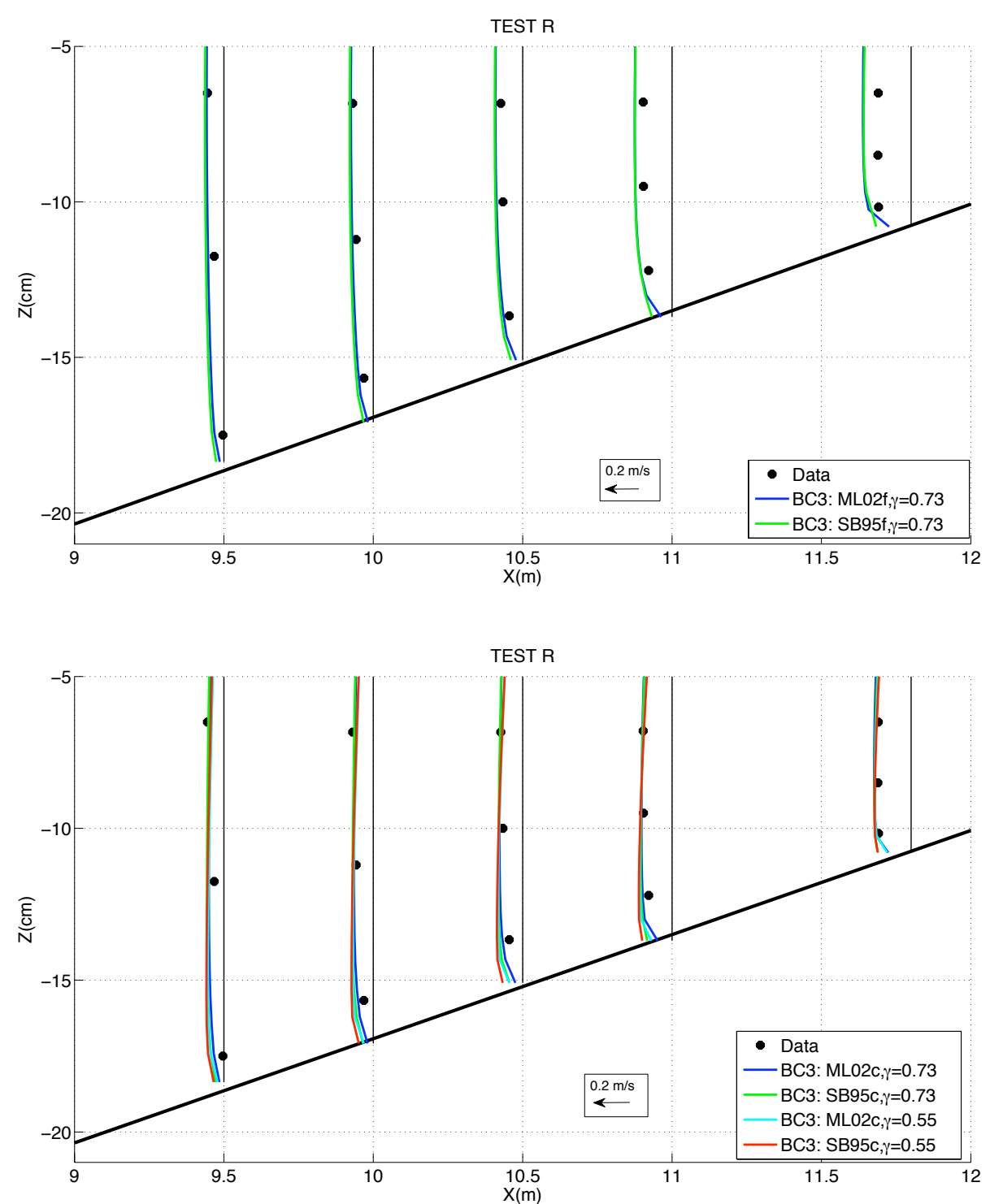

Figure 3.3: Comparison of some vertical profiles of the quasi-Eulerian cross-shore velocity. Black circles show data from Haas and Svendsen (2002). Top panel: One-way profiles. ML02 and SB95 results are shown in blue and green solid lines, respectively. Bottom panel: Two-way profiles. For $\gamma=0.73$, ML02 and SB95 results are shown in blue and green solid lines, respectively. For $\gamma=0.55$, they are in light blue and red solid lines, respectively. Bathymetry is plotted with a bold black line.

(Battjes and Janssen, 1978) are employed to artificially modify the shape and the intensity of the wave height. As expected, the $\gamma$ modify the profiles (see Figure 3.4): the breaking point is shifted, with a breaking event that appears sooner for $\gamma=0.55$ (in comparison with $\gamma=0.73$ ), with more dissipation after breaking. Moreover the largest shoal is produced for $\gamma=0.73$. At a given $\gamma$ value, the feedback causes an 
additional shoal (see Figure 3.4). When an opposite current is present, the dissipation of the wave energy due to breaking is increased and some parameterizations including this effect have been developped and tested (e.g. der Westhuysen (2012) and Dodet et al. (2013)). Here, the well-known parameterization of Battjes and Janssen (1978) is used. The wave height might be larger than expected because of this effect (see Figure 3.4, the red and green lines). However, as no measurements are available for shoal and our results fit rather well with the others measurements, the parameterization of der Westhuysen (2012) has not been implemented here. No blocking occurs because the maximum value for the ratio of the depth-integrated cross-shore velocity to the intrinsic wave group velocity (computed by the wave model) is about -0.1 in the rip channel instead of -1 . That confirms the conclusions of Özkan-Haller and Haller (2002) showing that wave blocking by rips is fairly rare. For a one-way coupling, the significant wave height is independent of the bottom stress parameterization because the current effects on the waves are not included in the numerical simulations. Therefore, equivalent results are obtained with the ML02 and SB95 parameterizations (see Figure 3.4, ML02f and SB95f). The best fit with the laboratory data is found for a two-way coupling with $\gamma=0.73$ (see Figure 3.4, red and green solid lines).

The feedback slightly influences the shape of the mean sea surface elevation (hereafter MSSE) (see Figure 3.5). The gradient of the MSSE, near the shore, is found the highest for simulations without the feedback, with a difference of about $10 \%$ in comparison with the two-way results (see Figure 3.6). These conclusions are true for all bottom stress parameterizations.

The depth induced breaking constant modulates the shape of the MSSE which is correctly simulated for $\gamma=0.73$. When $\gamma=0.55$ is used, the shape is smoothed, the setdown is weaker and the setup event appears sooner in comparison with $\gamma=0.73$ 473 (see Figure 3.5). The cross-shore profiles of the significant wave height (see Figure 474 3) are in agreement with these conclusions, with a smaller shoal and a breaking 


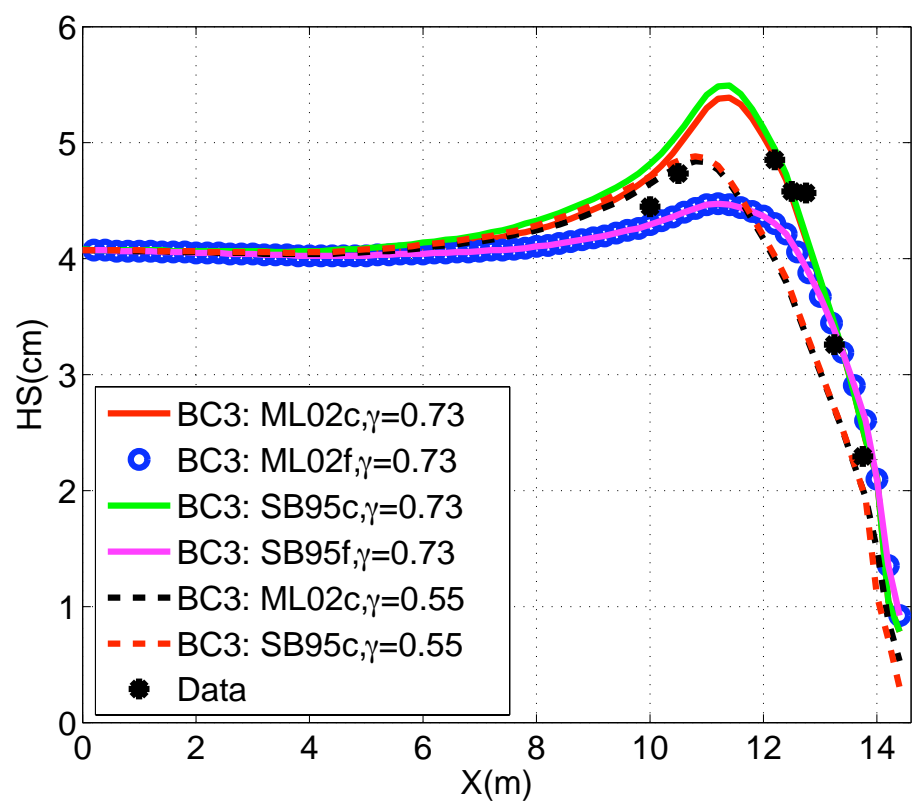

Figure 3.4: Cross-shore profiles of the significant wave height inside the rip channel. ML02c and ML02f: two-way and one-way simulations with ML02, respectively. SB95c and SB95f: two-way and one-way simulations with SB95, respectively. Data: data from the Haas and Svendsen (2002) experiment. The $\gamma=0.55$ and $\gamma=0.73$ labels refer to a depth induced breaking constant set to 0.55 and 0.73 , respectively.

event which appeared sooner for $\gamma=0.55$. Onshore, the cross-shore gradient of the two-way MSSE computed with the mixing parameterization (ML02) is increased by about $50 \%$ from $\gamma=0.55$ to $\gamma=0.73$. It is caused by an increasing of the bottom shear stress of about $50 \%$ when ML02 is used. That is coherent because $\gamma$ influences the mixing due to wave breaking which is directly included in ML02. SB95 being based on the near-bottom wave orbital velocity, it is less sensitive to the mixing than ML02. $\gamma$ has a little impact on the near-bottom cross-shore velocity except near the shore where the depth is very shallower and the undertow is predominant (see Figure 3.7). The bottom shear stress produced by $\gamma=0.73$ is the strongest which is coherent because the highest shoal is obtained for this value of $\gamma$ (see Figure 3.4).

The two parameterizations correctly simulated the shape of the MSSE. The crossshore gradient of the MSS is modified by the parameterization, in particular near 
the shore. An increasing of $12 \%$ is observed for all cases by the use of ML02 instead of SB95. The near-bottom cross-shore velocity is reduced when ML02 is used. The main peak is decreased by about 30\% with ML02 in comparison with SB95 which is caused by an increasing of the bottom shear stress of about $40 \%$ (see Figure 3.7) knowing that the growth is the strongest for the two-way simulations. Near the shore, the decreasing of the ML02 velocities, due to an increasing of the bottom stress (of about $40 \%$ ), is the origin of the $12 \%$ on the gradient of the MSSE.

We conclude that: (a) the simulated wave set-up is dependent on: -the bottom stress formulation, -coupling mode, -the depth-induced breaking constant (b) the feedback has little impact on the shape of the MSSE but increases the gradient of the MSSE near the shore (c) the use of the turbulent quantities in the parameterization of the bottom shear stress is a relevant option for future numerical investigation of the wave set-up. A variation of $12 \%$ is found between the ML02 and SB95 configurations. However, a strong dependence to the $\gamma$ value being also found, the 2 parameterization of the dissipation of the wave energy by breaking also appears as 3 a key point to improve the wave set-up simulations. 


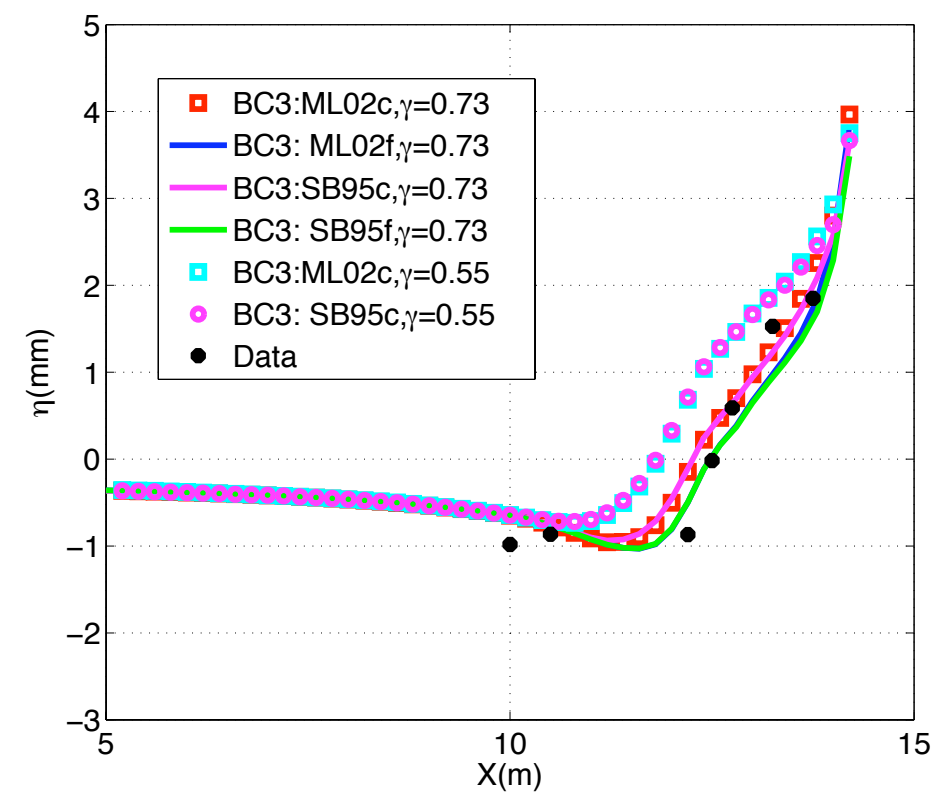

Figure 3.5: Cross-shore profiles of the mean sea surface elevation. ML02c and ML02f: two-way and one-way simulations with ML02, respectively. SB95c and SB95f: twoway and one-way simulations with SB95, respectively. Data: data from the Haas and Svendsen (2002) experiment. The $\gamma=0.55$ and $\gamma=0.73$ labels refer to a depth induced breaking constant set to 0.55 and 0.73 , respectively.

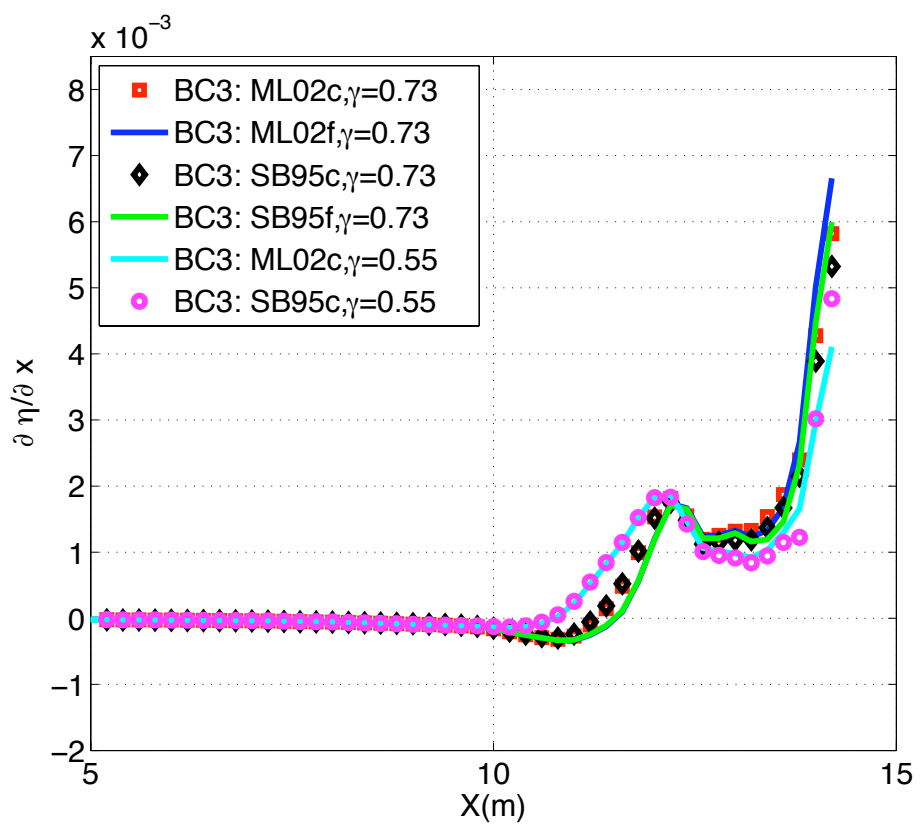

Figure 3.6: Cross-shore profiles of the cross-shore gradient of the mean sea surface elevation. Same labels as previously. 

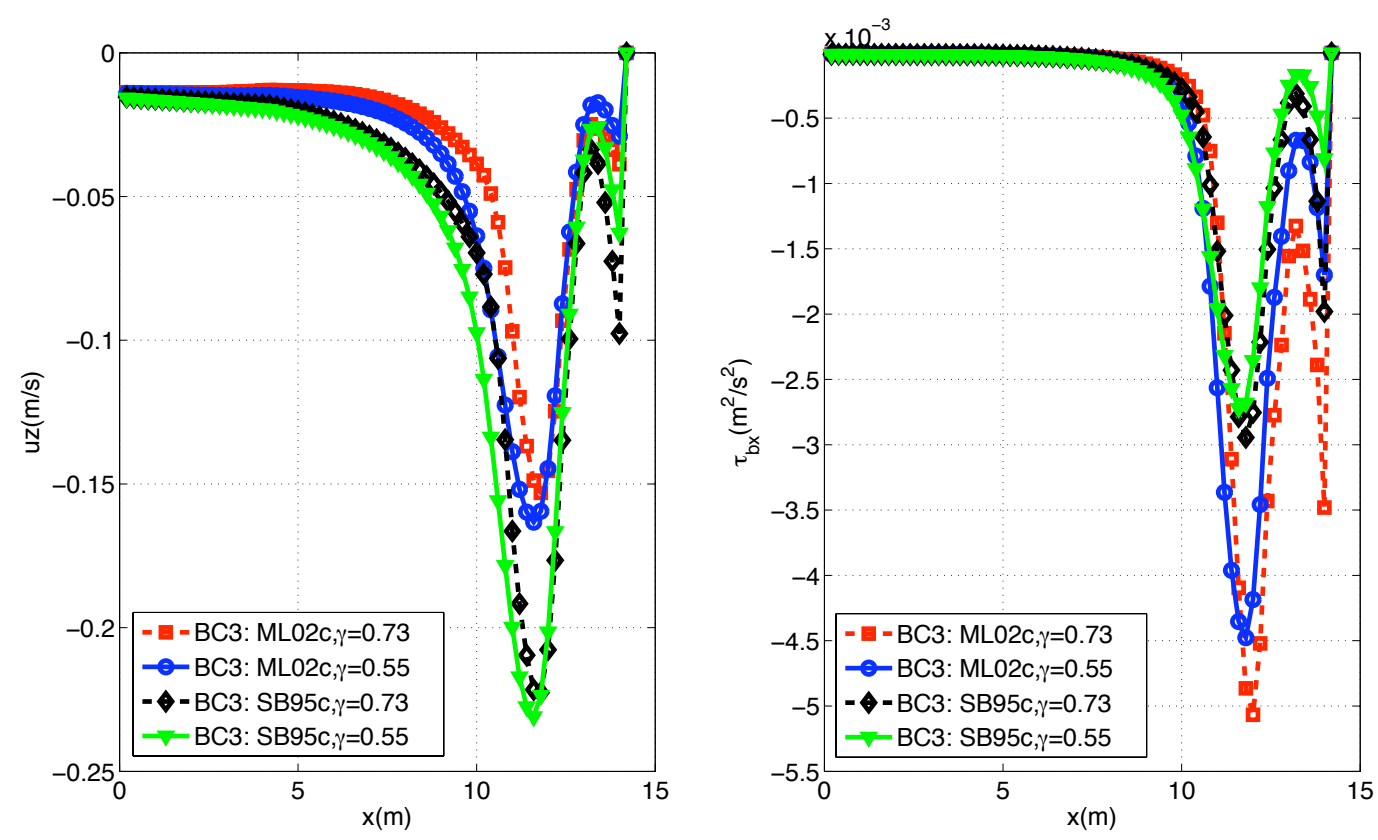

Figure 3.7: Cross-shore profiles of: -the near-bottom quasi-Eulerian cross-shore velocity (left row), -the x-component of the bottom stress (right row). Two-way profiles for the mixing (ML02c) and Soulsby (SB95c) parameterizations are shown. Two values of $\gamma$ are tested: $\gamma=0.55$ and $\gamma=0.73$.

\section{Summary and conclusions}

Numerical investigations using the mixing parameterization described within the scope of this paper have been conducted. Two studies are carried out. First, a onedimensional study allowed us to assess the performance of ML02 and adapt it at our modeling system. Second, a nearshore study allowed us to highlight the impact of the mixing parameterization (ML02) on the simulation of the wave set-up, in comparison with the one of Soulsby (1995).

The one-dimensional vertical study shows the strong dependence of the results on the $F_{2 z}$ function. This function impacts the magnitude and shape of the vertical velocity profile. We show that $F_{2 z}$ depends on both $z_{\text {bot }}$ and the near-bottom wave orbital velocity. This function was developped by Mellor in 2002 to fit a phase-resolving velocity and must be tuned to be used on another modeling situation. Therefore, 
a new function, $F_{2 z, \text { mod }}$, has been derived. The velocity profiles agree with the phase-resolving ones. In contrast, near-bottom TKE is overestimated because of the intrinsic formulation of the mixing parameterization that uses an additional source of TKE to account for oscillations of the wave bottom boundary layer. We show that $F_{2 z, \text { mod }}$ works well with a refined mesh at high resolution but also with a regular mesh at low resolution.

Wave breaking does not modify significantly the vertical profile of velocity. The most significant impact is obtained at low resolution with a one-meter depth. Wave breaking reduces the near-surface velocity and increases the turbulent quantities near the surface. At high resolution, two characteristic lengths were tested to distribute the wave breaking sources over depth. They led to almost similar results, knowing that some differences arose from the alteration of the vertical discretization near both the bottom and the surface. The TKE budget depends on the characteristic length but the production terms balance the dissipation and diffusion terms in all cases. On the whole, the mixing parameterization shows good performance in presence of wave breaking.

Then, in a nearshore study, we performed several tests against the laboratory data of Haas and Svendsen (2002). Comparisons with SB95 are also carried out. The vertical structure of the rip current agrees with the description given by Kumar et al. (2012): the velocity is maximum within the water column and decreases towards the surface and the bottom. Observational data may suggest another shape but, unfortunately, without surface values to enable a thorough comparison with our numerical results. Qualitatively, the modeled velocity agrees with the observations, with an RMS error of about $4 \%$ for TEST R, in a two-way mode. We show that the vertical profiles located near the shore are highly sensitive to the coupling mode: the feedback appears to be necessary to fit observations. Both parameterizations produce similar vertical profiles of velocity except near the bottom. The best results are obtained by the mixing parameterization used in a two-way coupling mode. Next 
to the bottom, the cross-shore velocity is strongly impacted by the bottom shear stress parameterization. A reduction of $30 \%$ for the rip velocity is observed with ML02 in comparison with SB95.

We find that the wave set-up is modulated by the bottom shear stress parameterization, the coupling mode and the depth-induced breaking constant. An increasing of $12 \%$ is obtained with ML02 in comparison with SB95. This is caused by a bottom stress which is increased of about $40 \%$. The coupling mode also impacts the gradient of MSSE: the wave set-up is reduced by $10 \%$ percents when the feedback is activated. The mixing parameterization is highly sensitive the value of the $\gamma$. As a result, between simulations using $\gamma=0.55$ and $\gamma=0.73$, an increasing of $50 \%$ is observed with ML02 because of the bottom shear stress growth. Taking mixing into account in the bottom stress parameterization seems to be a promising way to improve the numerical simulation of the wave set-up. However, our study highlights the difficulty to use of the ML02 mixing parameterization because of its lack of universality caused by the $F_{2 z}$ function. Therefore, the use of another parameterization also based on turbulent quantities may be profitable to improve the simulation of the wave set-up. As this type of parameterization appears to be highly sensitive to $\gamma$, an additional work on the dissipation of the wave energy by wave breaking, in presence of opposite currents, would be suitable.

A generalized parameterization of the vertical mixing in association with bottom friction could be developped in a near future by updating first the vertical profiles that were proposed by Mellor (2002) and should be compared to measured turbulence properties in surf zones. Some tests could be performed for energetic wave conditions like in Apostos et al. (2007). 
578

\section{Appendix}

\section{A Some vertical meshes}

The discrete vertical distribution for the terrain-following coordinate $(\varsigma)$ has the generic form:

$$
\begin{array}{ll}
\varsigma=\frac{\exp \left(a_{1} \cdot \lambda\right)}{a_{3}}-a_{2}, & \varsigma<\lambda_{\max } / 2, \\
\varsigma=\frac{-\exp \left(a_{1} \cdot\left(-\lambda+\lambda_{\max }\right)\right)}{a_{3}}+a_{4}, & \varsigma \geq \lambda_{\max } / 2 .
\end{array}
$$

where $\lambda_{\max }$ is the total number of grid points, set here to $1200 . \lambda$ represents the vertical grid index and the value of the coefficients for each mesh is given in the following table :

\begin{tabular}{|c|c|c|c|c|c|c|}
\hline & $\mathbf{a}_{\mathbf{1}}$ & $\mathbf{a}_{\mathbf{2}}$ & $\mathbf{a}_{\mathbf{3}}$ & $\mathbf{a}_{\mathbf{4}}$ & $\mathbf{z}_{\text {bot }}(\mathbf{m})$ & $\mathrm{F}_{\mathbf{2 z}}^{\mathbf{b o t}}$ \\
\hline${\text { Mesh } \mathrm{n}^{\circ} \mathbf{1}}$ & $1.26 .10^{-3}$ & 1.42 & $0.23 .10^{1}$ & $4.30 .10^{-1}$ & $3.00 .10^{-2}$ & 0.20 \\
\hline Mesh $^{\circ} \mathbf{2}$ & $1.26 .10^{-2}$ & 0.99 & $3.98 .10^{3}$ & $5.00 .10^{-3}$ & $9.20 .10^{-2}$ & 0.90 \\
\hline${\text { Mesh }{ }^{\circ} \mathbf{3}}^{3}$ & $3.00 .10^{-2}$ & 1.00 & $1.28 .10^{8}$ & 0.00 & $3.20 .10^{-8}$ & 5.50 \\
\hline Mesh n $^{\circ} \mathbf{4}$ & $2.00 .10^{-2}$ & 1.00 & $3.20 .10^{5}$ & 0.00 & $1.30 .10^{-5}$ & 2.40 \\
\hline${\text { Mesh }{ }^{\circ} \mathbf{5}}^{1.70 .10^{-2}}$ & 0.99 & $7.49 .10^{3}$ & 0.00 & $7.60 .10^{-5}$ & 1.80 \\
\hline
\end{tabular}

The elevation $(z)$ from the bottom is given by: $z=2 h \varsigma+2 h$.

The $F_{2 z}$ function is given in Mellor (2002) (see his equation (21a)):

$$
\begin{aligned}
F_{2 z}= & -0.0488+0.02917 l z+0.01703 l z^{2} \\
& +\left[1.125\left(l z_{0}+5\right)+0.125\left(l z_{0}+5\right)^{4}\right] \\
& \times\left(-0.0102-0.00253 l z+0.00273 l z^{2}\right),
\end{aligned}
$$


$584 \quad$ with $l z=\ln \left(\frac{z \omega}{\left|\mathbf{u}_{\mathbf{b}}\right|}\right)$ and $l z_{0}=\log _{10}\left(\frac{z_{0} \omega}{\left|\mathbf{u}_{\mathbf{b}}\right|}\right)$.

585

\section{Acknowledgments}

The authors thank K. Haas for the laboratory data and for his advice. We thank the anonymous reviewers for their useful comments. A-C. Bennis acknowledges the support of a post-doctoral grant from Université de Bretagne Occidentale, the PREVIMER and IOWAGA projects. F. Dumas is supported by the PREVIMER project. F. Ardhuin is supported by a FP7-ERC grant \#240009 "IOWAGA". B. Blanke is supported by the Centre National de la Recherche Scientifique.

\section{References}

Alves, J. H. G. M. and M. L. Banner, 2003: Performance of a saturation-based dissipation-rate source term in modeling the fetch-limited evolution of wind waves. J. Phys. Oceanogr., 33, 1274-1298.

Apotsos, A., B. Raubenheimer, S. Elgar, R. T. Guza, and J. A. Smith, 2007: Effects of wave rollers and bottom stress on wave setup. J. Geophys. Res., 112, C02 003, doi:10.1029/2006JC003549.

Ardhuin, F., N. Rascle, and K. A. Belibassakis, 2008: Explicit wave-averaged primitive equations using a generalized Lagrangian mean. Ocean Modelling, 20, 35-60, doi:10.1016/j.ocemod.2007.07.001.

Barrantes, A. I. and O. S. Madsen, 2000: Near-bottom flow and flow resistance for currents obliquely incident to two-dimensional roughness elements. J. Geophys. Res., 105 (C11), 26 253-26264.

Battjes, J. A., 1975: Modeling of turbulence in surf zone. Symposium on Modeling Techniques, 1051-1061. 
Battjes, J. A. and J. P. F. M. Janssen, 1978: Energy loss and set-up due to breaking of random waves. Proceedings of the 16th international conference on coastal engineering, ASCE, 569-587.

Belcher, S. E. and J. C. R. Hunt, 1993: Turbulent shear flow over slowly moving waves. J. Fluid Mech., 251, 109-148.

Bennis, A.-C., F. Ardhuin, and F. Dumas, 2011: On the coupling of wave and three-dimensional circulation models: Choice of theoretical framework, practical implementation and adiabatic tests. Ocean Modelling, 40, 260-272.

Bourchtein, A. and L. Bourchtein, 2006: Modified time splitting scheme for shallow water equations. Mathematics and Computers in Simulation, 73, 52-64.

Bowen, A. J., 1969: The generation of longshore currents on a plane beach. J. Mar. Res., 27, 206-215.

Burchard, H., 2001: Simulating the wave-enhanced layer under breaking surface waves with two-equation turbulence models. J. Phys. Oceanogr., 31, 3133-3145.

Craig, P. D. and M. L. Banner, 1994: Modeling wave-enhanced turbulence in the ocean surface layer. J. Phys. Oceanogr., 24, 2546-2559.

der Westhuysen, A. J. V., 2012: Spectral modeling of wave dissipation on negative current gradients. Coastal Eng., 68, 17-30.

Dodet, G., X. Bertin, N. Bruneau, A. B. Fortunato, A. Nahon, and A. Roland, 2013: Wave-current interactions in a wave-dominated tidal inlet. J. Geophys. Res., 118, $1587-1605$.

Eldeberky, Y. and J. A. Battjes, 1996: Spectral modelling of wave breaking: Application to boussinesq equations. J. Geophys. Res., 101, 1253-1264. 
Feddersen, F., E. L. Gallagher, R. T. Guza, and S. E. Feddersen, 2003: The drag coefficient, bottom roughness, and wave-breaking in the nearshore. Coastal Eng., 48, 189-195.

Feddersen, F., R. T. Guza, S. Elgar, and T. H. C. Herbers, 2000: Velocity moments in alongshore bottom stress parameterizations. J. Geophys. Res., 105 (C4), 86738686.

Feddersen, F. and J. H. Trowbridge, 2005: The effect of wave breaking on surf-zone turbulence and alongshore currents: a modelling study. J. Phys. Oceanogr., 35, $2187-2204$

Filipot, J.-F., F. Ardhuin, and A. Babanin, 2010: A unified deep-to-shallow-water spectral wave breaking dissipation formulation. Part 1. Breaking probability. $J$. Geophys. Res., 115, C04022.

Haas, K. A. and I. A. Svendsen, 2002: Laboratory measurements of the vertical structure of rip currents. J. Geophys. Res., 107 (C5), 3047.

Haller, M. C., R. A. Dalrymple, and I. A. Svendsen, 2002: Experimental study of nearshore dynamics on a barred beach with rip channels. J. Geophys. Res., 107 (C6), 3061.

Jensen, B. L., B. M. Sumer, and J. Fredsøe, 1989: Turbulent oscillatory boundary layers at high Reynolds numbers. J. Fluid Mech., 206, 265-297.

Kantha, L. H. and C. A. Clayson, 2004: On the effect of surface gravity waves on mixing in the oceanic mixed layer. Ocean Modelling, 6, 101-124.

Kumar, N., G. Voulgaris, J. C. Warner, and M. Olabarrieta, 2012: Implementation of the vortex force formalism in the coupled ocean-atmosphere-wave-sediment transport (COAWST) modeling system for inner shelf and surf zone applications. Ocean Modelling, 47, 65-95. 
Lazure, P. and F. Dumas, 2008: An external-internal mode coupling for a 3d hydrodynamical model for applications at regional scale (MARS). Adv. Water Resources, 31, 233-250.

Leckler, F., F. Ardhuin, J.-F. Filipot, and A. Mironov, 2013: Dissipation source terms and whitecap statistics. Ocean Modelling, in press.

Longuet-Higgins, M. S., 1970: Longshore currents generated by obliquely incident sea waves, 2. J. Geophys. Res., 75, 6790-6801.

Longuet-Higgins, M. S. and R. W. Stewart, 1963: A note on wave set-up. J. Mar. Res., 21, 4-10.

McWilliams, J. C., J. M. Restrepo, and E. M. Lane, 2004: An asymptotic theory for the interaction of waves and currents in coastal waters. J. Fluid Mech., 511, $135-178$.

Mellor, G., 2002: Oscillatory bottom boundary layers. J. Phys. Oceanogr., 32, 30753088.

Miles, J., 1996: Surface wave generation: a viscoelastic model. J. Fluid Mech., 322, $131-145$.

Moghimi, S., K. Klingbeil, U. Grawe, and H. Burchard, 2012: A direct comparison of the depth-dependent radiation stress method and a vortex force formulation within a three-dimensional ocean model. Ocean Modelling, 1-38.

Nelson, R. C., 1994: Depth limited wave heights in very flat regions. Coastal Eng., 23, 43-59.

Nelson, R. C., 1997: Heights limits in top down and bottom up wave environments. Coastal Eng., 32, 247-254. 
Özkan-Haller, H. T. and M. C. Haller, 2002: Wave breaking and rip current circulation. Proceedings of the 28th International Conference on Coastal Engineering, Cardiff, UK, 705-717.

Rascle, N., B. Chapron, F. Ardhuin, and A. Soloviev, 2013: A note on the direct injection of turbulence by breaking waves. Ocean Modelling, 70, 145-151.

Raubenheimer, B., R. T. Guza, and S. Elgar, 2001: Field observations of wave-driven setdown and setup. J. Geophys. Res., 106 (C3), 4629-4638.

Reniers, A. J. H. M., A. R. van Dongeren, J. A. Battjes, and E. B. Thornton, 2002: Linear modeling of infragravity waves during delilah. J. Geophys. Res., 107 (C10), 3137, doi:10.1029/2001JC001083.

Soulsby, R. L., 1995: Bed shear stresses due to combined waves and currents. In: Stive, M., Fredsøe, J., Hamm, L., Soulsby, R., Teisson, C., Winterwerp, J. (Eds). Advances in Coastal Morphodynamics, Delft Hydraulics, Delft, The Netherlands, $420-423$.

Svendsen, I., 2006: Introduction to nearshore hydrodynamics. World Scientific, Singapore, $722 \mathrm{pp}$.

Terray, E. A., M. A. Donelan, Y. C. Agrawal, W. M. Drennan, K. K. Kahma, A. J. Williams, P. A. Hwang, and S. A. Kitaigorodskii, 1996: Estimates of kinetic energy dissipation under breaking waves. J. Phys. Oceanogr., 26, 792-807.

Walstra, D. J. R., J. Roelvink, and J. Groeneweg, 2000: Calculation of wave-driven currents in a 3D mean flow model. Proceedings of the 27th international conference on coastal engineering, Sydney, ASCE, Vol. 2, 1050-1063.

Weir, B., Y. Uchiyama, E. M. Lane, J. M. Restrepo, and J. C. McWilliams, 2011: A vortex force analysis of the interaction of rip currents and surface gravity waves. J. Geophys. Res., 116, C05001. 
${ }_{703} \mathrm{Yu}$, J. and D. N. Slinn, 2003: Effects of wave-current interaction on rip currents. J. $704 \quad$ Geophys. Res., 108 (C3), 3088. 